TRANSACTIONS OF THE

AMERICAN MATHEMATICAL SOCIETY

Volume 358, Number 9, September 2006, Pages 3937-3967

S 0002-9947(05)03788-8

Article electronically published on August 1, 2005

\title{
TOPOLOGICAL OBSTRUCTIONS TO CERTAIN LIE GROUP ACTIONS ON MANIFOLDS
}

\author{
PISHENG DING
}

\begin{abstract}
Given a smooth closed $S^{1}$-manifold $M$, this article studies the extent to which certain numbers of the form $\left(f^{*}(x) \cdot P \cdot C\right)[M]$ are determined by the fixed-point set $M^{S^{1}}$, where $f: M \rightarrow K\left(\pi_{1}(M), 1\right)$ classifies the universal cover of $M, x \in H^{*}\left(\pi_{1}(M) ; \mathbb{Q}\right), P$ is a polynomial in the Pontrjagin classes of $M$, and $C$ is in the subalgebra of $H^{*}(M ; \mathbb{Q})$ generated by $H^{2}(M ; \mathbb{Q})$. When $M^{S^{1}}=\varnothing$, various vanishing theorems follow, giving obstructions to certain fixed-point-free actions. For example, if a fixed-point-free $S^{1}$-action extends to an action by some semisimple compact Lie group $G$, then $\left(f^{*}(x) \cdot P \cdot C\right)[M]=0$. Similar vanishing results are obtained for spin manifolds admitting certain $S^{1}$-actions.
\end{abstract}

\section{INTRODUCTION}

Terminological convention. Throughout this article, unless indicated otherwise, "Lie group" and "G" signify "positive-dimensional connected compact Lie group", "manifold" and " $M$ " signify "oriented closed smooth manifold", and, "action" signifies "smooth action".

Given a manifold $M$, let $\pi$ denote $\pi_{1}(M)$ and let $f: M \rightarrow K(\pi, 1)$ classify the universal covering space of $M$; let $C$ be an element of the subalgebra of $H^{*}(M ; \mathbb{Q})$ generated by $H^{2}(M ; \mathbb{Q})$, and let $P$ be a rational polynomial in the Pontrjagin classes of $M$.

Suppose $M$ is an $S^{1}$-manifold. Let $o: S^{1} \rightarrow M$ be the orbit of some basepoint in $M$. As a standard fact, $o_{*}\left(\pi_{1}\left(S^{1}\right)\right)$ is a central subgroup of $\pi$. Let $\pi^{\prime}=$ $\pi / o_{*}\left(\pi_{1}\left(S^{1}\right)\right)$, and let $\rho: \pi \rightarrow \pi^{\prime}$ be the quotient map. Let $x \in H^{*}(\pi ; \mathbb{Q})$, $y \in H^{*}\left(\pi^{\prime} ; \mathbb{Q}\right)$.

This article mainly studies the extent to which certain numbers of the form $\left(f^{*}(x) \cdot P \cdot C\right)[M]$ are determined by the fixed-point set $M^{S^{1}}$. When $M^{S^{1}}=\varnothing$, various vanishing theorems follow, giving obstructions to certain fixed-point-free actions. My investigation draws upon the Atiyah-Singer's index theory (mainly the $G$-signature theorem), some equivariant bundle theory of [8], and [3]'s work relating an effective $G$-action on $M$ to $\pi_{1}(M)$.

Received by the editors October 9, 2003 and, in revised form, June 9, 2004.

2000 Mathematics Subject Classification. Primary 57S15, 58J20; Secondary 57R91, 57R20.

Key words and phrases. Group actions, circle actions, characteristic numbers, index theory, $G$-signature theorem, geometric topology.

The author thanks his Ph.D. advisor, Sylvain Cappell, for pointing out the general direction along which the results of this article are developed. The author is grateful to the referee for many constructive suggestions.

(C)2005 American Mathematical Society Reverts to public domain 28 years from publication 
In this Introduction, a number of salient results of this work are described along with a review of some related existing results. I first give an overview of several vanishing statements, which form various parts of Theorem A. The proof of parts of Theorem A relies in an essential way on some localization results stated in Theorem $\mathrm{C}$, the exposition of which forms a major portion of this article. I mention in Theorem D two vanishing propositions about certain spin $S^{1}$-manifolds of a similar flavor. Theorem B provides a digression related to Theorem $\mathrm{A}\left(3^{\prime}\right)$.

Using the then newly invented index theory, 2] obtains "residue formulae" for the Pontrjagin numbers of $M$ in terms of the submanifold $M^{S^{1}} \hookrightarrow M$ and the $S^{1}$-action on its normal bundle; this in particular implies that, when $M^{S^{1}}=\varnothing$, all the Pontrjagin numbers of $M$ vanish. Combining this result and [3]'s work, one immediately obtains

Theorem A(1). Suppose $M^{S^{1}}=\varnothing$. Then, $\left(f^{*} \rho^{*}(y) \cdot P\right)[M]=0$; if, in addition, $\operatorname{Im}\left(\pi_{1}\left(S^{1}\right) \stackrel{o_{*}}{\rightarrow} \pi_{1}(M)\right)$ is finite, then $\left(f^{*}(x) \cdot P\right)[M]=0$.

Suppose $M^{S^{1}}=\varnothing$. Using differential-geometric method, [12] shows that, if $H^{1}(M)=\{0\}$, then $C[M]=0$; using index theory and some equivariant bundle theory of [8], [5] shows that, if $\pi_{1}(M)$ is finite, then $\left(c^{k} \cdot \mathbf{L}(M)\right)[M]=0$ where $c \in H^{2}(M ; \mathbb{Q})$ and $\mathbf{L}(M)$ is the Hirzebruch $L$-class of $M$. Generalizing these two propositions is

Theorem A(2). Suppose $M^{S^{1}}=\varnothing$. If $H^{1}(M)=\{0\}$, then $\left(f^{*} \rho^{*}(y) \cdot P \cdot C\right)[M]$ $=0$; if, in addition, $\operatorname{Im}\left(\pi_{1}\left(S^{1}\right) \stackrel{o_{*}}{\rightarrow} \pi_{1}(M)\right)$ is finite, then $\left(f^{*}(x) \cdot P \cdot C\right)[M]=0$.

We show in the next result that, under some hypothesis different from that in Theorem $\mathrm{A}(2)$, the same conclusion can be drawn.

Theorem A(3). Suppose $M^{S^{1}}=\varnothing$. If the $S^{1}$-action on $M$ extends to an action by a semisimple Lie group $G$, then $\left(f^{*}(x) \cdot P \cdot C\right)[M]=0$.

Thus, the nonvanishing of any number of the form $\left(f^{*}(x) \cdot P \cdot C\right)[M]$ is an obstruction to extending any fixed-point-free $S^{1}$-action to an action by a semisimple Lie group $G$. Said differently, this yields

Theorem $\mathbf{A}\left(\mathbf{3}^{\prime}\right)$. If a semisimple Lie group $G$ acts on $M$ with some $g \in G$ acting freely, then $\left(f^{*}(x) \cdot P \cdot C\right)[M]=0$.

Note that, if $f^{*}(x)\left[M^{m}\right]=0$ for all $x \in H^{*}(\pi ; \mathbb{Q})$, then $f_{*}\left[M^{m}\right]=0 \in$ $H_{m}(\pi ; \mathbb{Q})$. Hence, Theorem $\mathrm{A}\left(3^{\prime}\right)$ implies that a nonzero $f_{*}[M]$ is an obstruction to certain $G$-actions with $G$ semisimple. In fact, a stronger statement holds, showing that a nonzero $f_{*}[M]$ is an obstruction to any $G$-action with $G$ semisimple. I mention two earlier results along this direction.

[4] shows that, if $M$ is aspherical, then the only Lie groups acting effectively on $M$ are tori with dimensions bounded above by $\operatorname{rank} \mathcal{Z}(\pi)$ ( " $\mathcal{Z}$ " signifying "the center of"), and such effective torus-actions must be fixed-point-free and have only finite isotropy subgroups. [12 shows that, if there exist $\omega_{1}, \cdots, \omega_{m} \in H^{1}(M)$ such that $\prod_{j} \omega_{j}\left[M^{m}\right] \neq 0$, then the only Lie groups acting effectively on $M$ are tori. Note that weaker than the hypotheses of both of these two statements is the condition that $f_{*}[M] \neq 0$; thus the following theorem generalizes them. 
Theorem B. Suppose $M^{m}$ satisfies the condition that $f_{*}[M] \neq 0 \in H_{m}(\pi ; \mathbb{Q})$.

(1) If $G$ is semisimple, then any $G$-action on $M$ is trivial.

(2) If $G$ acts effectively on $M$, then $G$ is a torus with $\operatorname{dim} G \leq \operatorname{rank} \mathcal{Z}(\pi)$ and all isotropy subgroups are finite.

(3) If $G$ acts nontrivially on $M$, then $M^{S}=\varnothing$ for some circle subgroup $S \subset G$; hence, Theorems $\mathrm{A}(1)$ and $\mathrm{A}(2)$ apply.

The core of the proof of Theorems A(2) and A(3) is some "localization analysis" which the next theorem deals with. Given an $S^{1}$-manifold $M$, Theorem $\mathrm{C}$ treats "localization" of $(P \cdot C)[M]$, i.e., determination of such numbers in terms of fixedpoint data. Let $R=\left(c^{k} \cdot P\right)[M]$ where $c \in \operatorname{Im}\left(H^{2}(M) \rightarrow H^{2}(M ; \mathbb{Q})\right)$. It turns out, as a matter of algebra, that to compute $(P \cdot C)[M]$, it suffices to only compute $R$. Therefore, the next theorem is only stated for $R$.

Let $\mathcal{L}(c)$ be the complex line bundle over $M$ whose first Chern class is $c$. Call the linear $S^{1}$-action on a fiber of the normal bundle over $M^{S^{1}}$ an isotropy $S^{1}$ representation.

Theorem C. Let $M$ be a $S^{1}$-manifold.

(1) If $H^{1}(M)=0$, or, if the $S^{1}$-action extends to a $G$-action with $\pi_{1}(G)=\{0\}$, then $R$ can be determined in terms of: the submanifold $M^{S^{1}} \hookrightarrow M$, the isotropy $S^{1}$-representations, and the restriction to $\left.\mathcal{L}(c)\right|_{M^{S^{1}}}$ of any lifted $S^{1}$-action on $\mathcal{L}(c)$.

(2) If, for some semisimple Lie group $G$, the $S^{1}$-action extends to a $G$-action with each component of $M^{S^{1}}$ containing a point of $M^{G}$, then $R$ can be determined in terms of the submanifold $M^{S^{1}} \hookrightarrow M$ and the isotropy $S^{1}$ representations.

(3) Let $F_{0}$ be the union of those components of $M^{S^{1}}$ with codimensions congruent to 0 mod 4. Then, under the same condition as in $(2),(\mathbf{L}(M) \cdot C)[M]$ can be determined in terms of the submanifold $F_{0} \hookrightarrow M$ (and is independent of the isotropy $S^{1}$-representations). In particular, if, in addition to the stated condition, $F_{0}=\varnothing$, then $(\mathbf{L}(M) \cdot C)[M]=0$.

(The condition on the extension of the $S^{1}$-action to some $G$-action appearing in Theorem $\mathrm{C}$ is discussed in the Remark below.)

Suppose $M$ is a spin manifold admitting a nontrivial $S^{1}$-action. 1] shows that

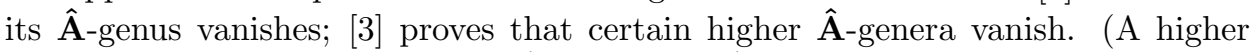
$\hat{\mathbf{A}}$-genus is a number of the form $\left(f^{*}(x) \cdot \hat{\mathbf{A}}(M)\right)[M]$.) I show

Theorem D. Let $M$ be a spin manifold admitting a nontrivial $S^{1}$-action which extends to a $G$-action with $G$ a semisimple Lie group.

(1) If each component of $M^{S^{1}}$ contains a point of $M^{G}$, then $(\hat{\mathbf{A}}(M) \cdot C)[M]=0$.

(2) If $M^{S^{1}}=M^{G}$, then $\left(f^{*}(x) \cdot \hat{\mathbf{A}}(M) \cdot C\right)[M]=0$.

Remark. The condition that for some semisimple $G$ the $S^{1}$-action extends to a $G$-action with each component of $M^{S^{1}}$ containing a point of $M^{G}$ is essential for the validity of parts of Theorems A, C and D. For example, realizing $S^{2}$ as the homogeneous space $S U(2) / S$ where $S \subset S U(2)$ is a maximal torus (which is a circle subgroup), one has a fixed-point-free $S U(2)$-action on $S^{2}$ but $\left(S^{2}\right)^{S}$ is nonempty; now there indeed are nonvanishing cohomology classes in $H^{2}\left(S^{2}\right)$, which 
invalidates the conclusions of Theorems $\mathrm{A}\left(3^{\prime}\right), \mathrm{C}(3)$ and $\mathrm{D}$. More generally, given a semisimple Lie group $G$ and a closed proper subgroup $H$ containing a maximal torus $T$ of $G, G / H$ is an even-dimensional manifold with a fixed-point-free $G$-action but $(G / H)^{T}$ is certainly nonempty; often then, the characteristic numbers appearing in Theorems $\mathrm{A}\left(3^{\prime}\right), \mathrm{C}(3)$ and $\mathrm{D}$ are nonzero.

Now we discuss how this condition might be realized. If $M$ admits a semifree $S U(2)$-action, then, for any circle subgroup $S \subset S U(2), M^{S}=M^{S U(2)}$; hence the condition is met. ( $S U(2)$ happens to be the only semisimple Lie group that can act semifreely on manifolds.)

In general, given a $G$-action, one may ask when there will be a circle subgroup $S \subset G$ with $M^{S}=M^{G}$.

For $T$ a maximal torus in $G$, there is a circle subgroup $S \subset T$ with $M^{S}=M^{T}$; thus, any condition relating $M^{S}$ and $M^{G}$ can be reformulated as a condition relating $M^{T}$ and $M^{G}$. It is shown in [6] that an even-codimensional component $F$ of $M^{G}$ is also a component of $M^{T}$ if and only if the equivariant Euler class of the normal bundle of $F \hookrightarrow M$ satisfies some "invertibility" condition, which I now describe.

Let $F$ be a $2 k$-codimensional component of $M^{G}$ and $N(F) \rightarrow F$ the normal bundle of $F$ in $M$. The equivariant Euler class of $N(F)$, denoted by $e_{G}(N(F))$, is an element of

$$
H^{2 k}\left(E G \times_{G} F ; \mathbb{Q}\right)=H^{2 k}(B G \times F ; \mathbb{Q}) \simeq \bigoplus_{j=0}^{k} H^{2 k-2 j}(B G ; \mathbb{Q}) \otimes H^{2 j}(F ; \mathbb{Q}) .
$$

So we may write

$$
e_{G}(N(F))=\alpha_{2 k}(F) \otimes 1+\sum \alpha \otimes \beta
$$

where $\alpha_{2 k}(F) \in H^{2 k}(B G ; \mathbb{Q}), \alpha \in H^{2(k-j)}(B G ; \mathbb{Q})$ and $\beta \in H^{2 j}(F ; \mathbb{Q})$ with $j>0$. [6] shows that $F$ is also a connected component of $M^{T}$ if and only if $\alpha_{2 k}(F) \neq 0$. Hence, if every component of $M^{G}$ satisfies this condition and if $M^{T}$ has no more components than $M^{G}$, then $M^{G}=M^{T}$.

\section{Preliminaries}

The purpose of this section is three-fold: to review some existing results, to deduce some immediate consequences of them relevant to our developments, and in doing so, to establish notation and terminology.

2.1. Equivariant bundle theory. We review the problem of lifting a group action on $X$ to an action on the total space of a bundle over $X$. We will be primarily interested in the case of an $S^{1}$-bundle (or equivalently, a complex line bundle).

2.1.1. Lifting $G$-actions in fiber bundles. Let $X$ be a $G$-space and $E \stackrel{\operatorname{Pr}}{\rightarrow} X$ a principal $H$-bundle, $G$ and $H$ being Lie groups (not necessarily connected). Some natural questions arising from this situation are whether, when, and how the $G$ action on $X$ can be lifted to a $G$-action on $E$ commuting with the $H$-action on $E$. (The commutativity of the $G$-action and $H$-action on $E$ is equivalent to the condition that $G$ acts on $E$ by ( $H$-bundle)-isomorphisms, which is to be assumed whenever we speak of a lifted $G$-action on the total space of a principal $H$-bundle.) For example, when $G$ acts isometrically on a Riemannian manifold $M^{m}$, the principal $O(m)$-bundle $P$ associated with the Riemannian structure of $M^{m}$ always admits a natural lifting, the $G$-action on $P$ being induced by that on $M$. 
For a $G$-space $X$ having the homotopy type of a $\mathrm{CW}$-complex, let $\mathfrak{B}_{(G, H)}(X)$ be the set of equivalence classes of $G$-( $H$-bundle)s over $X$, a $G$-( $H$-bundle) over $X$ being a principal $H$-bundle whose total space admits a lifted $G$-action, and, two $G$-( $H$-bundle)s being equivalent if there is a $G$-equivariant ( $H$-bundle)-equivalence between them.

For a space $Y$ having the homotopy type of a $\mathrm{CW}$-complex, let $\mathfrak{B}_{H}(Y)$ be the set of equivalence classes of principal $H$-bundles over $Y$.

Given a $G$-space $X$, there is an obvious map

$$
\phi: \mathfrak{B}_{(G, H)}(X) \longrightarrow \mathfrak{B}_{H}(X)
$$

which forgets the $G$-equivariant structure, yielding the underlining $H$-bundle of a $G$-(H-bundle). $\operatorname{Im} \phi$ is precisely the set of equivalence classes of $H$-bundles which admit liftings of the $G$-action on $X$. Let $X_{G}=E G \times_{G} X$. Choosing a basepoint in $E G$ gives rise to an inclusion $i: X \rightarrow X_{G}$, which induces a restriction map:

$$
i^{*}: \mathfrak{B}_{H}\left(X_{G}\right) \longrightarrow \mathfrak{B}_{H}(X) \text {. }
$$

Given a $G$-(H-bundle) $E \stackrel{\operatorname{Pr}}{\rightarrow} X$, the Borel construction gives $E_{G} \stackrel{\operatorname{Pr}_{G}}{\longrightarrow} X_{G}$, an $H$ bundle over $X_{G}$. This produces a map

$$
\psi: \mathfrak{B}_{(G, H)}(X) \longrightarrow \mathfrak{B}_{H}\left(X_{G}\right) \text {. }
$$

Evidently, $i^{*} \circ \psi=\phi$.

The following proposition is shown in [8]:

Proposition 2.1 ([8]). When $H$ is abelian, $\psi$ is a bijection and hence $\operatorname{Im} i^{*}=\operatorname{Im} \phi$.

2.1.2. Lifting connected group actions in $S^{1}$-bundles. We now specialize the result in Proposition 2.1 to the case in which $H=S^{1}$ and $G$ is connected. Identifying an $S^{1}$-bundle with its first Chern class, we have

$$
\mathfrak{B}_{S^{1}}(Y) \simeq\left[Y, B S^{1}\right] \simeq H^{2}(Y) \text {. }
$$

By Proposition 2.1, $\operatorname{Im} i^{*}=\operatorname{Im} \phi$. We may regard $i^{*}$ as the restriction

$$
i^{*}: H^{2}\left(X_{G}\right) \longrightarrow H^{2}(X),
$$

the image of which can readily be studied by the Leray-Serre spectral sequence associated with the fiber bundle $X \stackrel{i}{\hookrightarrow} X_{G} \stackrel{p}{\rightarrow} B G$. As $\pi_{1}(B G) \simeq \pi_{0}(G)=\{0\}$, this bundle has a simple system of local coefficients on the base $B G$.

Corollary 2.2. Let $G$ be a Lie group acting on a space $X$. Suppose $H^{3}(B G)=\{0\}$ and $H^{1}(X)=\{0\}$. Let $E \rightarrow X$ be an $S^{1}$-bundle. Then $E \rightarrow X$ can be given $a$ $G$-equivariant structure, i.e., the $G$-action on $X$ can be lifted to a $G$-action on $E$. Furthermore, the set of equivalence classes of the $G$ - $\left(S^{1}\right.$-bundle)s resulting from all possible liftings is in bijective correspondence with $H^{2}(B G)$.

Proof. In the fiber bundle $X \stackrel{i}{\hookrightarrow} X_{G} \stackrel{p}{\rightarrow} B G, H^{1}(B G)=\{0\}$ by simple connectivity of $B G$ (which is equivalent to the connectivity of $G$ ), and, $H^{1}(X)=\{0\}$ by hypothesis; thus we have the following exact sequence, which is a portion of the Serre exact sequence derived from the Serre spectral sequence:

$$
\{0\}=H^{1}(X) \longrightarrow H^{2}(B G) \stackrel{p^{*}}{\longrightarrow} H^{2}\left(X_{G}\right) \stackrel{i^{*}}{\longrightarrow} H^{2}(X) \longrightarrow H^{3}(B G)=\{0\} ;
$$

see [10]. By the exactness of this sequence, $i^{*}$ is surjective; hence so is $\phi$, proving the first statement. Since $i^{*} \circ \psi=\phi$ and $\psi$ is a bijection, the multitude of liftings 
(i.e., the multitude of $\phi^{-1}[E]$ ) is the same as that of $\operatorname{ker} i^{*}=\operatorname{Im} p^{*} \simeq H^{2}(B G)$, as claimed.

Corollary 2.3. Let $G$ be a simply connected Lie group acting on a space $X$. Then every $S^{1}$-bundle over $X$ admits a unique $G$-equivariant structure, i.e., $\phi$ : $\mathfrak{B}_{\left(G, S^{1}\right)}(X) \rightarrow \mathfrak{B}_{S^{1}}(X)$ is an isomorphism.

Proof. If $G$ is 1-connected, $G$ is then also 2-connected as $\pi_{2}(G)=\{0\}$ for any compact Lie group. Thus, for $1 \leq j \leq 3, \pi_{j}(B G) \simeq \pi_{j-1}(G)=\{0\}$, and by the Hurewicz theorem, $H^{j}(B G)=\{0\}$. Under these conditions, we have the following exact sequence, which is a portion of the Serre exact sequence:

$$
\{0\}=H^{2}(B G) \stackrel{p^{*}}{\longrightarrow} H^{2}\left(X_{G}\right) \stackrel{i^{*}}{\longrightarrow} H^{2}(X) \longrightarrow H^{3}(B G)=\{0\},
$$

implying that $i^{*}: H^{2}\left(X_{G}\right) \rightarrow H^{2}(X)$ is an isomorphism. As $i^{*} \circ \psi=\phi, \phi$ : $\mathfrak{B}_{\left(G, S^{1}\right)}(X) \rightarrow \mathfrak{B}_{S^{1}}(X)$ is an isomorphism, as claimed.

Next, we make an observation regarding $E^{G}$, the fixed-point set of the total space of a $G$-( $S^{1}$-bundle). As above, let $X$ be a $G$-space and $\operatorname{Pr}: E \rightarrow X$ be an $S^{1}$-principal bundle. As we see in the above discussion, liftings, if they exist, may not be unique. In general, the lifted $G$-action on the fiber $\operatorname{Pr}^{-1}[a]$ over a fixed point $a \in X^{G}$ depends on the global lifting and may not always be the trivial one. However, when $G$ is semisimple, the next proposition shows that any lifted $G$-action on $\operatorname{Pr}^{-1}[a]$ for $a \in X^{G}$ is always the trivial action.

Proposition 2.4. Let $G$ be a semisimple Lie group acting on a space $X$ and let $\operatorname{Pr}: E \rightarrow X$ be a $G-\left(S^{1}\right.$-bundle $)$. Then, $E^{G}=\operatorname{Pr}^{-1}\left[X^{G}\right]$.

Proof. Over each connected component $X_{\nu}^{G}$ of $X^{G}$, the $G$-action on $\operatorname{Pr}^{-1}\left[X_{\nu}^{G}\right]$ produces a 1-dimensional complex representation $\rho_{\nu}: G \rightarrow S^{1}$. It suffices to show that there is no nontrivial Lie group homomorphism $\rho: G \rightarrow S^{1}$, i.e., that $G$ has no connected abelian quotient, which follows immediately from the Lie-algebratheoretic definition of semisimplicity.

Remark 2.5. A principal $S^{1}$-bundle $E$ over $X$ is the "same" as a complex line bundle $\mathcal{L} \rightarrow X$, and, a lifted $G$-action on $\mathcal{L}$ is simply a homomorphism $G \rightarrow \operatorname{Iso}(\mathcal{L})$ with a prescribed $G$-action on the zero section of $\mathcal{L}$. The results in this section can then be restated in terms of complex line bundles.

2.2. The $G$-signature and $G$-spin theorems. We review those parts of the index theory that are related to the present study. We first briefly describe the construction of the twisted $G$-signature of a $G$-manifold, then state the AtiyahSinger's $G$-signature formula, and finally include a description of the $G$-spinorindex.

2.2.1. The $G$-signature. Let $M^{2 n}$ be a Riemannian manifold and $G$ be a Lie group acting on $M$ by orientation-preserving isometries. Let $\Lambda^{*}\left(T^{*} \otimes \mathbb{C}\right)$ be the bundle of complex exterior algebras of the complexified cotangent bundle. There is a bundle homomorphism $\tau: \Lambda^{k}\left(T^{*} \otimes \mathbb{C}\right) \rightarrow \Lambda^{2 n-k}\left(T^{*} \otimes \mathbb{C}\right)$ defined via the Hodge star $*$ :

$$
\tau(a)=i^{k(k-1)+n} *(a) \text { for } a \in \Lambda^{k}\left(T^{*} \otimes \mathbb{C}\right) .
$$

Note that $\tau^{2}$ is the identity. For $E \rightarrow M$ a $G$-equivariant complex vector bundle, let $\Omega_{E}^{*}(M)$ be the space of complex differential forms with coefficients in $E$, i.e., 
the space of smooth sections of $\Lambda^{*}\left(T^{*} \otimes \mathbb{C}\right) \otimes E$. $\tau$ canonically induces an operator $\tau_{E}$ on $\Omega_{E}^{*}(M) . \tau_{E}$ is an involution. Let $\Omega_{E}^{ \pm}(M)$ be the \pm 1 -eigenspaces of $\tau_{E}$. Let

$$
D=d+d^{*}: \Omega^{*}(M) \longrightarrow \Omega^{*}(M),
$$

where $d$ is the de Rham operator and $d^{*}$ its formal adjoint (with respect to the inner product on $\Omega^{*}$ induced by the Riemannian metric). Upon equipping $E$ with a connection, $D$ induces an operator $D_{E}$ on $\Omega_{E}^{*}(M)$ and $D_{E}$ anticommutes with $\tau_{E}$. Let $D_{E}^{+}: \Omega_{E}^{+}(M) \rightarrow \Omega_{E}^{-}(M)$ be the restriction of $D_{E}$ to $\Omega_{E}^{+}(M)$. Then the twisted $G$-signature, $\operatorname{Sign}_{G}(M ; E)$, is defined to be the $G$-index of $D_{E}^{+}$:

$$
\operatorname{ind}_{G}\left(D_{E}^{+}\right)=\left[\operatorname{ker} D_{E}^{+}\right]-\left[\operatorname{coker} D_{E}^{+}\right] \in R(G) .
$$

This index invariant of $D_{E}^{+}$is independent of the choice of connections on $E$, which justifies our notational suppression of the chosen connection. Regarding elements of $R(G)$ as character functions $G \rightarrow \mathbb{C}$, we denote the value of $\operatorname{Sign}_{G}(M ; E)$ at $g \in G$ by $\operatorname{Sign}_{G}(M ; E)(g)$.

Index theory gives a localization formula for $\operatorname{Sign}_{G}(M ; E)(g)$, i.e., a formula in terms of the topology of $M^{g}$, the $g$-action on the normal bundle $N$ of $M^{g}$, the bundle $\left.E\right|_{M^{g}}$ and the $g$-action on $\left.E\right|_{M^{g}}$. We now describe this formula in the special case where $g \in G$ is a topological generator of a toral subgroup $T \subset G$.

As $g$ generates $T, M^{g}=M^{T}$. Hence $\operatorname{Sign}_{G}(M ; E)(g)$ depends only on the $T$ action, i.e.,

$$
\operatorname{Sign}_{G}(M ; E)(g)=\operatorname{Sign}_{T}(M ; E)(g) .
$$

$E$ enters the formula via a class $\operatorname{ch}\left(\left.E\right|_{M^{g}}\right)(g) \in H^{*}\left(M^{g} ; \mathbb{C}\right)$, which we now describe. As $M^{g}$ is a trivial $T$-space, we can identify $K_{T}\left(M^{g}\right)$ with $K\left(M^{g}\right) \otimes R(T)$ and define $\operatorname{ch}()(g): K_{T}\left(M^{g}\right) \rightarrow H^{*}\left(M^{g} ; \mathbb{C}\right)$ as the map

$$
\operatorname{ch} \otimes \chi(g): K\left(M^{g}\right) \otimes R(T) \longrightarrow H^{*}\left(M^{g} ; \mathbb{C}\right) \otimes \mathbb{C} \simeq H^{*}\left(M^{g} ; \mathbb{C}\right) .
$$

Over each $x \in M^{g}$, the fiber $N_{x}$ of $N$ is a real $T$-module, which can be decomposed into a sum of two-dimensional irreducible subrepresentations, each of which is of the following type and hence has a canonical complex structure:

$$
g \mapsto\left(\begin{array}{cc}
\cos \theta & -\sin \theta \\
\sin \theta & \cos \theta
\end{array}\right), \quad \text { or } \quad g \mapsto e^{i \theta}, \quad \theta \in(0, \pi) .
$$

For $\theta \in(0, \pi)$, let $N_{x}(\theta)$ be the sum of those irreducible subrepresentations given by $g \mapsto e^{i \theta}$. Then,

$$
N_{x}=\bigoplus_{\theta \in(0, \pi)} N_{x}(\theta),
$$

where each $N_{x}(\theta)$ has a canonical complex structure. (There are only finitely many $\theta$ for which $N_{x}(\theta)$ is nontrivial.) Let $M^{g}=\bigcup_{\nu} M_{\nu}^{g}$, where $M_{\nu}^{g}$ are the connected components of $M^{g}$, and let $N_{\nu}$ denote the normal bundle of $M_{\nu}^{g}$. The fiberwise decomposition leads to a bundle decomposition

$$
N_{\nu}=\bigoplus_{\theta \in(0, \pi)} N_{\nu}(\theta) .
$$

The canonical orientation of $N_{\nu}$ induced by the complex structure gives an orientation of $M_{\nu}^{g}$ such that $T\left(M_{\nu}^{g}\right) \oplus N_{\nu}=\left.(T M)\right|_{M_{\nu}^{g}}$. 
For a manifold $X^{m}$, the Hirzebruch $\hat{\mathbf{L}}$-class, denoted by $\hat{\mathbf{L}}(X)$, is defined by

$$
\hat{\mathbf{L}}(X)=\prod_{j=1}^{\left[\frac{m+1}{2}\right]} \frac{x_{j} / 2}{\tanh \left(x_{j} / 2\right)}
$$

where $\left\{+x_{j},-x_{j} \mid 1 \leq j \leq\left[\frac{m+1}{2}\right]\right\}$ are the formal Chern roots of either $T X^{m} \otimes \mathbb{C}$ or $\left(T X^{m} \oplus \mathbf{1}_{\mathbb{R}}\right) \otimes \mathbb{C}$ according to whether $m$ is even or odd respectively. We write $\hat{L}_{k}(X)$ for the $4 k$-dimensional component of $\hat{\mathbf{L}}(X)$.

We are now ready to write the Atiyah-Singer's $G$-signature formula for our case.

Theorem 2.6 (G-Signature Theorem; 2]). For g a generator of a torus in $G$,

$$
\begin{aligned}
& \operatorname{Sign}_{G}\left(M^{2 n} ; E\right)(g) \\
& =\sum_{\nu}\left\{2^{t_{\nu}} \operatorname{ch}\left(\left.E\right|_{M_{\nu}^{g}}\right)(g) \hat{\mathbf{L}}\left(M_{\nu}^{g}\right) \prod_{\theta \in(0, \pi)} \prod_{j=1}^{d_{\nu}(\theta)} \operatorname{coth} \frac{x_{\nu, j}(\theta)+i \theta}{2}\right\}\left[M_{\nu}^{g}\right]
\end{aligned}
$$

where $t_{\nu}=\frac{1}{2} \operatorname{dim} M_{\nu}^{g}, d_{\nu}(\theta)=\operatorname{dim}_{\mathbb{C}} N_{\nu}(\theta)$, and $x_{\nu, j}(\theta), 1 \leq j \leq d_{\nu}(\theta)$, are the formal Chern roots of the complex bundle $N_{\nu}(\theta)$.

In this formula, the product over $\theta \in(0, \pi)$ is a finite product, as $d_{\nu}(\theta) \neq 0$ for only finitely many values of $\theta$.

The $G$-index of $D_{E}^{+}$evaluated at $e \in G$ is just the ordinary index of $D_{E}^{+}$:

$$
\text { ind }\left(D_{E}^{+}\right)=\operatorname{dim} \operatorname{ker} D_{E}^{+}-\operatorname{dim} \operatorname{coker} D_{E}^{+} \text {. }
$$

We denote ind $\left(D_{E}^{+}\right)$by $\operatorname{Sign}(M ; E)$. Index theory provides the following formula.

Theorem 2.7 ([2]).

$$
\begin{aligned}
\operatorname{Sign}_{G}\left(M^{2 n} ; E\right)(e) & =\operatorname{Sign}\left(M^{2 n} ; E\right) \\
& =2^{n}(\operatorname{ch}(E) \hat{\mathbf{L}}(M))[M] .
\end{aligned}
$$

2.2.2. The $G$-spinor-index. For $M^{2 n}$ a spin $G$-manifold (i.e., a spin manifold endowed with a $G$-action preserving the spin structure) and $E$ a complex $G$-bundle over $M$, 2 constructs an elliptic operator called the Dirac operator whose $G$-index is called the $G$-spinor-index and is denoted by $\operatorname{Spin}_{G}(M ; E)$. The value of the $G$-spinor-index at $e \in G$ is just the ordinary index of the Dirac operator and is denoted by $\operatorname{Spin}(M ; E)$.

The $G$-spin theorem, similar to the $G$-signature theorem, gives a localization formula for $\operatorname{Spin}_{G}(M ; E)(g)$ in terms of the fixed-point data of the $g$-action. We now describe this result.

For a manifold $X^{m}$, the $\hat{\mathbf{A}}$-class, denoted by $\hat{\mathbf{A}}(X)$, is defined by

$$
\hat{\mathbf{A}}(X)=\prod_{j=1}^{\left[\frac{\left.\frac{m+1}{2}\right]}{2}\right.} \frac{x_{j} / 2}{\sinh \left(x_{j} / 2\right)}
$$

where $\left\{+x_{j},-x_{j} \mid 1 \leq j \leq\left[\frac{m+1}{2}\right]\right\}$ are the formal Chern roots of either $T X^{m} \otimes \mathbb{C}$ or $\left(T X^{m} \oplus \mathbf{1}_{\mathbb{R}}\right) \otimes \mathbb{C}$ according to whether $m$ is even or odd respectively. We write $\hat{A}_{k}(X)$ for the $4 k$-dimensional component of $\hat{\mathbf{A}}(X)$.

Using the same notation and making the same assumptions as in the discussion of the $G$-signature theorem, we state the $G$-spin theorem. 
Theorem 2.8 (G-Spin Theorem; 1], [2]). For g a generator of a torus in $G$,

$$
\begin{aligned}
& \operatorname{Spin}_{G}\left(M^{2 n} ; E\right)(g) \\
& =\sum_{\nu}\left\{\sigma_{\nu} \operatorname{ch}\left(\left.E\right|_{M_{\nu}^{g}}\right)(g) \hat{\mathbf{A}}\left(M_{\nu}^{g}\right) \prod_{\theta \in(0, \pi)} \prod_{j=1}^{d_{\nu}(\theta)} \frac{e^{\frac{1}{2}\left(i \theta-x_{\nu, j}(\theta)\right)}}{1-e^{\left(i \theta-x_{\nu, j}(\theta)\right)}}\right\}\left[M_{\nu}^{g}\right]
\end{aligned}
$$

where for each $\nu, \sigma_{\nu}$ equals either 1 or -1 and depends on the action of $g$ on the principal Spin $(2 n)$-bundle associated with the spin-structure of $M$.

Theorem 2.9 ([2]).

$$
\begin{aligned}
\operatorname{Spin}_{G}\left(M^{2 n} ; E\right)(e) & =\operatorname{Spin}\left(M^{2 n} ; E\right) \\
& =2^{n}(\operatorname{ch}(E) \hat{\mathbf{A}}(M))[M] .
\end{aligned}
$$

\section{LOCALIZATION THEOREMS ON CERTAIN $G$-MANIFOLDS}

In this section, we obtain some localization results for certain "characteristic numbers" of some $G$-manifolds. Suppose $M$ is an $S^{1}$-manifold. Under certain conditions, we study, in $\S 3.1$, the dependence of $(\hat{\mathbf{L}}(M) \cdot C)[M]$ on $M^{S^{1}}$. Under less restrictive conditions, we then study, in $\S 3.2$, more general numbers of the form $(P \cdot C)[M]$.

3.1. $(\hat{\mathbf{L}}(M) \cdot C)[M]$. We begin with a theorem; the condition on the fixed point set appearing therein is remarked upon in $\S 1$.

Theorem 3.1. Let $G$ be semisimple and $M^{2 n}$ a G-manifold. Suppose there exists a circle subgroup $S \subset G$ such that every component of $M^{S}$ intersects $M^{G}$. Let $F_{0}$ be the union of those components of $M^{S}$ with codimensions congruent to $0 \bmod 4$, and let $i_{0}: F_{0} \hookrightarrow M$ be the inclusion map. Then $(\hat{\mathbf{L}}(M) \cdot C)[M]$ is determined by the submanifold $i_{0}: F_{0} \hookrightarrow M$ (and is independent of the $S$-action around $F_{0}$.) To be precise, this number can be computed in terms of $i_{0}^{*}(C), \hat{\mathbf{L}}\left(F_{0}\right)$ and $\left[F_{0}\right]$ using formulae (3.3) and (3.4) and Lemma 3.4. In particular, if, in addition to the stated condition, $F_{0}=\varnothing$, then $(\hat{\mathbf{L}}(M) \cdot C)[M]=0$.

Remark 3.2. To simplify the proof of this and some later results, we make several observations:

(1) By compactness of $G$, a $G$-manifold $M$ can always be endowed with a Riemannian structure so that the $G$-action on $M$ is isometric. The tool of index theory is then readily applicable.

(2) If $G$ is a semisimple compact Lie group acting on $M$, then, $\widetilde{G}$, the compact universal cover of $G$, acts on $M$ in a canonical way: $\widetilde{G} \rightarrow G \rightarrow \operatorname{Diff}(M)$. And there exists a circle subgroup $\widetilde{S} \subset \widetilde{G}$ with $M^{S}=M^{\widetilde{S}}$. Therefore, for the proof of Theorem 3.1, there is no loss of generality in assuming that $G$ is simply connected.

Proof of Theorem 3.1. By Remark 3.2, we assume that $G$ is simply connected and $G$ acts isometrically on $M$. The $G$-action preserves the orientation of $M$, as $G$ is connected. To avoid triviality, we assume that the $S$-action on $M$ is nontrivial. 
Let $c \in \operatorname{Im}\left(H^{2}(M) \rightarrow H^{2}(M ; \mathbb{Q})\right)$. We first give a localization formula for rational numbers of the form

$$
\left(\hat{\mathbf{L}}(M) \cdot c^{d}\right)[M]
$$

in terms of the submanifold $F_{0}$.

Let $\mathcal{L}(c) \rightarrow M$ be the complex line bundle whose first Chern class is $c$. As $G$ is now assumed to be simply connected, by Corollary 2.3 , the $G$-action on $M$ admits a (unique) lifting to a $G$-action on $\mathcal{L}(c)$, turning $\mathcal{L}(c)$ into a $G$-equivariant complex line bundle. Thus, $\operatorname{Sign}_{G}(M ; \mathcal{L}(c))$, the $\mathcal{L}(c)$-twisted $G$-signature of $M$, is defined. Using complex coordinates $z$ (with $|z|=1$ ) on the circle subgroup $S$, we first develop a formula for $\operatorname{Sign}_{G}(M ; \mathcal{L}(c))(z) \in \mathbb{C}$. As $z \in S, \operatorname{Sign}_{G}(M ; \mathcal{L}(c))(z)=$ $\operatorname{Sign}_{S}(M ; \mathcal{L}(c))(z)$ when $M$ is regarded as an $S$-manifold.

In the following, we use the notation established in $\S 2.2 .1$. Let $z=e^{i \alpha}$ topologically generate $S$. Certainly, $M^{z}=M^{S}$. By choosing suitable orientations, the irreducible subrepresentations of the real $S$-module $N_{x}\left(x \in M^{S}\right)$ are of the form

$$
z \mapsto\left(\begin{array}{cc}
\cos k \alpha & -\sin k \alpha \\
\sin k \alpha & \cos k \alpha
\end{array}\right), \quad \text { or } \quad z \mapsto z^{k}, \text { with } k \in \mathbb{N} .
$$

Let $M^{S}=\bigcup_{\nu} M_{\nu}^{S}$, where $M_{\nu}^{S}$ are the components of $M^{S}$, and let $N_{\nu}$ denote the normal bundle of $M_{\nu}^{S} \hookrightarrow M$. As in $\S 2.2 .1$, there is a complex bundle decomposition determined by the irreducible $S$-modules appearing in $\left(N_{\nu}\right)_{x}$ with $x \in M_{\nu}^{S}$ :

$$
N_{\nu}=\bigoplus_{k \in \mathbb{N}} N_{\nu}(k) .
$$

For $k \in \mathbb{N}$, let $d_{\nu}(k)=\operatorname{dim}_{\mathbb{C}} N_{\nu}(k), t_{\nu}=\frac{1}{2} \operatorname{dim} M_{\nu}^{S}$, and $x_{\nu, j}(k), j=1, \cdots, d_{\nu}(k)$, be the formal Chern roots of the complex bundle $N_{\nu}(k)$.

According to the $G$-signature theorem,

$$
\begin{aligned}
& \operatorname{Sign}_{G}(M ; \mathcal{L}(c))(z) \\
& =\operatorname{Sign}_{S}(M ; \mathcal{L}(c))(z) \\
& =\sum_{\nu}\left\{2^{t_{\nu}} \operatorname{ch}\left(\left.\mathcal{L}(c)\right|_{M_{\nu}^{S}}\right)(z) \hat{\mathbf{L}}\left(M_{\nu}^{S}\right) \prod_{k \in \mathbb{N}} \prod_{j=1}^{d_{\nu}(k)} \operatorname{coth} \frac{x_{\nu, j}(k)+i k \alpha}{2}\right\}\left[M_{\nu}^{S}\right] .
\end{aligned}
$$

Letting $s_{\nu}(z)$ denote the $\nu$ th summand, we have

$$
\operatorname{Sign}_{G}(M ; \mathcal{L}(c))(z)=\sum_{\nu} s_{\nu}(z) .
$$

We now seek to express $s_{\nu}(z)$ in terms of $z$ instead of $\alpha$.

For each component $M_{\nu}^{S}$, there is an integer $l_{\nu}$ such that, for $x \in M_{\nu}^{S}$, $z$ acts on the fiber $\mathcal{L}(c)_{x}$ via the complex representation $z \mapsto z^{l_{\nu}}$. Hence,

$$
\operatorname{ch}\left(\left.\mathcal{L}(c)\right|_{M_{\nu}^{S}}\right)(z)=z^{l_{\nu}} \cdot e^{i_{\nu}^{*}(c)}
$$

Next, observe that

$$
\begin{aligned}
\operatorname{coth} \frac{x_{\nu, j}(k)+i k \alpha}{2} & =\frac{e^{\frac{1}{2}\left(x_{\nu, j}(k)+i k \alpha\right)}+e^{-\frac{1}{2}\left(x_{\nu, j}(k)+i k \alpha\right)}}{e^{\frac{1}{2}\left(x_{\nu, j}(k)+i k \alpha\right)}-e^{-\frac{1}{2}\left(x_{\nu, j}(k)+i k \alpha\right)}} \\
& =\frac{z^{k}+e^{-x_{\nu, j}(k)}}{z^{k}-e^{-x_{\nu, j}(k)}} .
\end{aligned}
$$


Hence,

$$
\begin{aligned}
& \operatorname{Sign}_{S}(M ; \mathcal{L}(c))(z) \\
& =\sum_{\nu}\left\{2^{t_{\nu}} z^{l_{\nu}} e^{i_{\nu}^{*}(c)} \hat{\mathbf{L}}\left(M_{\nu}^{S}\right) \prod_{k \in \mathbb{N}}\left(\prod_{j=1}^{d_{\nu}(k)} \frac{z^{k}+e^{-x_{\nu, j}(k)}}{z^{k}-e^{-x_{\nu, j}(k)}}\right)\right\}\left[M_{\nu}^{S}\right] .
\end{aligned}
$$

So far, $s_{\nu}(z)$ is only defined for $z$ a topological generator of $S$. If we extend $s_{\nu}(z)$ to a meromorphic function on $\mathbb{C}$, it then has poles at some roots of unity (certainly at 1 ), as can be seen by expanding $\frac{z^{k}+e^{-x_{\nu, j}(k)}}{z^{k}-e^{-x_{\nu, j}(k)}}$ into a polynomial in $x_{\nu, j}(k)$ :

$$
\frac{z^{k}+e^{-x_{\nu, j}(k)}}{z^{k}-e^{-x_{\nu, j}(k)}}=\frac{z^{k}+1}{z^{k}-1}+\sum_{m=1}^{t_{\nu}}(-1)^{m} \frac{2 z^{k}}{\left(z^{k}-1\right)^{m+1}}\left(x_{\nu, j}(k)\right)^{m} .
$$

However, $\operatorname{Sign}_{S}(M ; \mathcal{L}(c))(z)$ is a well-defined complex function for all $z \in S$; indeed, since $\operatorname{Sign}_{S}(M ; \mathcal{L}(c)) \in R(S) \simeq R\left(S^{1}\right), \operatorname{Sign}_{S}(M ; \mathcal{L}(c))(z)$ is a Laurent polynomial in $z$ and hence extends to an analytic function $w(z)$ on $\mathbb{C} \backslash\{0\}$. In particular, $w(z)$ has no poles on the unit circle. Consequently, in $\sum_{\nu} s_{\nu}(z)$, cancellation of poles among the summands $s_{\nu}(z)$ must take place, implying the multitude of components in $M^{S}$ if $M^{S} \neq \varnothing$.

The discussion of the two preceding paragraphs (in which the semisimplicity of $G$ plays no role) applies regardless of whether $\mathcal{L}(c)$ is present or not. We summarize this discussion in a proposition about the fixed-point set of an even-dimensional $S^{1}$-manifold.

Proposition 3.3. Let $S^{1}$ act nontrivially on an even-dimensional manifold $M$. Then,

(1) The number of components of $M^{S^{1}}$ does not equal 1.

(2) Suppose that $M^{S^{1}}=\{a, b\}$, a two-point set. Then $T_{a} M$ and $T_{b} M$ are equivalent as $S^{1}$-representations, and $\operatorname{Sign}(M)=0$.

Proof. If $M^{S^{1}} \neq \varnothing$, the poles of $s_{\nu_{0}}(z)$ must be cancelled by those in $\sum_{\nu \neq \nu_{0}} s_{\nu}(z)$ and hence $M^{S^{1}}$ has more than one component, proving (1).

For (2), let $W_{\nu}=\left\{k \in \mathbb{N} \mid N_{\nu}(k) \neq\{0\}\right\}$ for $\nu=a, b$. Then the $S^{1}$-signature of $M$ is

$$
\begin{aligned}
& \operatorname{Sign}_{S^{1}}(M)(z) \\
& =\prod_{k \in W_{a}}\left(\frac{z^{k}+1}{z^{k}-1}\right)^{d_{a}(k)}[a]+\prod_{k \in W_{b}}\left(\frac{z^{k}+1}{z^{k}-1}\right)^{d_{b}(k)}[b] .
\end{aligned}
$$

For this to be analytic on $S^{1}$, the two summands must have common poles and hence $W_{a}=W_{b}$. Let $k \in W_{a}=W_{b}$. At each $k$ th roots of unity, the poles in the above two terms are of order $d_{a}(k)$ and $d_{b}(k)$ respectively. For the poles to cancel each other out, it must be that $d_{a}(k)=d_{b}(k)$ and one of the two points $a$ and $b$ receives the negative orientation. Hence, $\operatorname{Sign}(M)=\operatorname{Sign}_{S^{1}}(M)(z)=0$.

Continuation of proof of Theorem 3.1. We now study the Laurent polynomial $w(z)$ $=\operatorname{Sign}_{S}(M ; \mathcal{L}(c))(z)$ further. 
We claim that $l_{\nu}=0$ for all $\nu$. For $y \in M^{G}, \mathcal{L}(c)_{y}$ is a 1-dimensional complex $G$ module. By Proposition 2.4, the semisimplicity of $G$ implies that $\mathcal{L}(c)_{y}$ is a trivial $G$-module. Therefore, $S$ acts trivially on $\mathcal{L}(c)_{y}$. By hypothesis, each component $M_{\nu}^{S}$ of $M^{S}$ contains some $y \in M^{G}$. Hence, by discreteness of representations, $S$ acts trivially on $\mathcal{L}(c)_{x}$ for every $x \in M_{\nu}^{S}$, i.e., $l_{\nu}=0$.

Putting this into the formula for $s_{\nu}(z)$, we have

$$
s_{\nu}(z)=\left\{2^{t_{\nu}} e^{i_{\nu}^{*}(c)} \hat{\mathbf{L}}\left(M_{\nu}^{S}\right) \prod_{k \in \mathbb{N}}\left(\prod_{j=1}^{d_{\nu}(k)} \frac{z^{k}+e^{-x_{\nu, j}(k)}}{z^{k}-e^{-x_{\nu, j}(k)}}\right)\right\}\left[M_{\nu}^{S}\right] .
$$

Regarded as a function on the extended complex plane $\overline{\mathbb{C}}$, each $s_{\nu}(z)$ is finite at 0 and $\infty$, as is clear by taking $\lim s_{\nu}(z)$. Then so is the Laurent polynomial $w(z)=\sum_{\nu} s_{\nu}(z)$. A Laurent polynomial which is finite at 0 and $\infty$ must be a constant. We now examine this constant.

$$
\begin{aligned}
w(0) & =\lim _{z \rightarrow 0} \sum_{\nu} s_{\nu}(z) \\
& =\sum_{\nu}\left(2^{t_{\nu}} e^{i_{\nu}^{*}(c)} \hat{\mathbf{L}}\left(M_{\nu}^{S}\right) \prod_{k \in \mathbb{N}} \prod_{j=1}^{d_{\nu}(k)}(-1)\right)\left[M_{\nu}^{S}\right] \\
& =\sum_{\nu}(-1)^{n-t_{\nu}} 2^{t_{\nu}}\left(e^{i_{\nu}^{*}(c)} \hat{\mathbf{L}}\left(M_{\nu}^{S}\right)\right)\left[M_{\nu}^{S}\right],
\end{aligned}
$$

while

$$
\begin{aligned}
w(\infty) & =\lim _{z \rightarrow \infty} \sum_{\nu} s_{\nu}(z) \\
& =\sum_{\nu}\left(2^{t_{\nu}} e^{i_{\nu}^{*}(c)} \hat{\mathbf{L}}\left(M_{\nu}^{S}\right)\right)\left[M_{\nu}^{S}\right] .
\end{aligned}
$$

Equating $w(0)$ and $w(\infty)$ yields

$$
\sum_{\left(n-t_{\nu}\right) \equiv 1 \bmod 2}\left(2^{t_{\nu}} e^{i_{\nu}^{*}(c)} \hat{\mathbf{L}}\left(M_{\nu}^{S}\right)\right)\left[M_{\nu}^{S}\right]=0 .
$$

Hence,

$$
w(z) \equiv \sum_{\operatorname{codim} M_{\nu}^{S} \equiv 0 \bmod 4}\left(2^{t_{\nu}} e^{i_{\nu}^{*}(c)} \hat{\mathbf{L}}\left(M_{\nu}^{S}\right)\right)\left[M_{\nu}^{S}\right] .
$$

We conclude that

$$
\begin{aligned}
\operatorname{Sign}_{G}(M ; \mathcal{L}(c))(e) & =\operatorname{Sign}_{S}(M ; \mathcal{L}(c))(1) \\
& =\sum_{\operatorname{codim} M_{\nu}^{S} \equiv 0 \bmod 4}\left(2^{t_{\nu}} e^{i_{\nu}^{*}(c)} \hat{\mathbf{L}}\left(M_{\nu}^{S}\right)\right)\left[M_{\nu}^{S}\right] .
\end{aligned}
$$


Applying Theorem 2.7 to our case leads to

$$
\begin{aligned}
& \operatorname{Sign}_{G}(M ; \mathcal{L}(c))(e) \\
& =\operatorname{Sign}(M ; \mathcal{L}(c)) \\
& =2^{n}(\operatorname{ch}(\mathcal{L}(c)) \hat{\mathbf{L}}(M))[M] \\
& =2^{n}\left(e^{c} \cdot \hat{\mathbf{L}}(M)\right)[M] \\
& =2^{n}\left\{\left(\sum_{j=0}^{n} \frac{c^{j}}{j !}\right) \cdot\left(\sum_{i=0}^{[n / 2]} \hat{L}_{i}(M)\right)\right\}[M] .
\end{aligned}
$$

To obtain a formula for $\left(\hat{\mathbf{L}}(M) \cdot c^{d}\right)[M]$ from (3.1) and (3.2), we use a technique of [5]. First we consider the case where $n$ is odd; let $n=2 r+1$. Let $\lambda$ be an integer. (3.1) and (3.2) yield

$$
\begin{aligned}
& \left(\sum_{j=0}^{r} \frac{1}{(2 j+1) !}(\lambda c)^{2 j+1} \hat{L}_{r-j}(M)\right)\left[M^{4 r+2}\right] \\
& =2^{-n} \operatorname{Sign}\left(M^{2 n} ; \mathcal{L}(\lambda c)\right) \\
& =2^{-n} \sum_{\quad \operatorname{codim} M_{\nu}^{S} \equiv 0 \bmod 4}\left(2^{t_{\nu}} e^{i_{\nu}^{*}(\lambda c)} \hat{\mathbf{L}}\left(M_{\nu}^{S}\right)\right)\left[M_{\nu}^{S}\right] .
\end{aligned}
$$

By letting $\lambda=1,2, \cdots, r+1$, the above is a system of $(r+1)$ linear equations in the $(r+1)$ variables $\left(\frac{1}{(2 j+1) !} c^{2 j+1} \hat{L}_{r-j}(M)\right)[M], j=0,1, \cdots, r$. The coefficient matrix is

$$
A=\left(a_{i j}\right)_{i, j=1}^{r+1}=\left(i^{2 j-1}\right)_{i, j=1}^{r+1}
$$

and

$$
\begin{aligned}
\operatorname{det} A & =\operatorname{det}\left(\left(i^{2 j-1}\right)_{i, j=1}^{r+1}\right) \\
& =\operatorname{det}\left(i \cdot\left(i^{2}\right)^{j-1}\right) \\
& =[(r+1) !] \operatorname{det}\left(\left(i^{2}\right)^{j-1}\right) \\
& =[(r+1) !] \prod_{1 \leq \alpha<\beta \leq r+1}\left(\beta^{2}-\alpha^{2}\right) \\
& \neq 0 .
\end{aligned}
$$

Let $A^{-1}=\left(b_{i j}\right)_{i, j=1}^{r+1}$. Solving the linear system gives

$$
\begin{aligned}
& \left(c^{2 j+1} \hat{L}_{r-j}(M)\right)\left[M^{4 r+2}\right] \\
& =\frac{(2 j+1) !}{2^{4 r+2}} \sum_{\lambda=1}^{r+1}\left\{b_{(j+1), \lambda} \cdot \sum_{\operatorname{codim} M_{\nu}^{S} \equiv 0 \bmod 4}\left(2^{t_{\nu}} e^{i_{\nu}^{*}(\lambda c)} \hat{\mathbf{L}}\left(M_{\nu}^{S}\right)\right)\left[M_{\nu}^{S}\right]\right\} .
\end{aligned}
$$


The case where $n$ is even is similarly handled. Let $n=2 r$. Let $\lambda$ be an integer. (3.1) and (3.2) yield

$$
\begin{aligned}
& \left(\sum_{j=0}^{r} \frac{1}{(2 j) !}(\lambda c)^{2 j} \hat{L}_{r-j}(M)\right)\left[M^{4 r}\right] \\
& =2^{-n} \operatorname{Sign}\left(M^{2 n} ; \mathcal{L}(\lambda c)\right) \\
& =2^{-n} \sum_{\operatorname{codim} M_{\nu}^{S} \equiv 0 \bmod 4}\left(2^{t_{\nu}} e^{i_{\nu}^{*}(\lambda c)} \hat{\mathbf{L}}\left(M_{\nu}^{S}\right)\right)\left[M_{\nu}^{S}\right] .
\end{aligned}
$$

By letting $\lambda=1,2, \cdots, r+1$, the above is a system of $(r+1)$ linear equations in the $(r+1)$ variables $\left(\frac{1}{(2 j) !} c^{2 j} \hat{L}_{r-j}(M)\right)[M], j=0,1, \cdots, r$. The coefficient matrix is

$$
A=\left(a_{i j}\right)_{i, j=1}^{r+1}=\left(i^{2 j-2}\right)_{i, j=1}^{r+1}
$$

and

$$
\begin{aligned}
\operatorname{det} A & =\operatorname{det}\left(\left(i^{2 j-2}\right)_{i, j=1}^{r+1}\right) \\
& =\operatorname{det}\left(\left(i^{2}\right)^{j-1}\right) \\
& =\prod_{\substack{1 \leq \alpha<\beta \leq r+1\\
}}\left(\beta^{2}-\alpha^{2}\right) \\
& \neq 0 .
\end{aligned}
$$

Let $A^{-1}=\left(b_{i j}\right)_{i, j=1}^{r+1}$. Solving the linear system gives

$$
\begin{aligned}
& \left(c^{2 j} \hat{L}_{r-j}(M)\right)\left[M^{4 r}\right] \\
& =\frac{(2 j) !}{2^{4 r}} \sum_{\lambda=1}^{r+1}\left\{b_{(j+1), \lambda} \cdot \sum_{\operatorname{codim} M_{\nu}^{S} \equiv 0 \bmod 4}\left(2^{t_{\nu}} e^{i_{\nu}^{*}(\lambda c)} \hat{\mathbf{L}}\left(M_{\nu}^{S}\right)\right)\left[M_{\nu}^{S}\right]\right\} .
\end{aligned}
$$

For $c \in \operatorname{Im}\left(H^{2}(M) \rightarrow H^{2}(M ; \mathbb{Q})\right),(3.3)$ and (3.4) give localization formulae for the rational number

$$
\left(\hat{\mathbf{L}}(M) \cdot c^{d}\right)[M]
$$

in terms of the submanifold $F_{0} \subset M$ consisting of those components of $M^{S}$ with codimensions congruent to $0 \bmod 4$.

To finally conclude the proof, we need to allow product of various $c_{j} \in H^{2}(M ; \mathbb{Q})$ to occur in the formula. Consider

$$
\left(\hat{\mathbf{L}}(M) \cdot \prod_{j} c_{j}^{d_{j}}\right)[M] .
$$

Let $\sum_{j} d_{j}=d$. By Lemma 3.4 (stated below and to be established in $\S 3.3$ ), $\prod_{j} c_{j}^{d_{j}}=\sum_{i} r_{i} x_{i}^{d}$ for some $x_{i} \in H^{2}(M ; \mathbb{Q})$ and $r_{i} \in \mathbb{Q}$. Hence

$$
\begin{aligned}
& \left(\hat{\mathbf{L}}(M) \cdot \prod_{j} c_{j}^{d_{j}}\right)[M] \\
& =\sum_{i} r_{i}\left(\hat{\mathbf{L}}(M) \cdot x_{i}^{d}\right)[M] .
\end{aligned}
$$


Since each $\left(\hat{\mathbf{L}}(M) \cdot x_{i}^{d}\right)[M]$ is computable, using equations (3.3) and (3.4), in terms of the submanifold $F_{0} \subset M$ consisting of those components of $M^{S}$ with codimensions congruent to $0 \bmod 4$, so is $\left(\hat{\mathbf{L}}(M) \cdot \prod_{j} c_{j}^{d_{j}}\right)[M]$.

This concludes the proof of Theorem 3.1.

We now state the lemma we needed at the end of the proof of Theorem 3.1.

Lemma 3.4. Suppose $\mathcal{A}=\bigoplus_{i=0}^{\infty} A_{i}$ is a graded $\mathbb{k}$-algebra, $\mathbb{k}$ being a field of characteristic 0 . Let l be a given positive integer, let $\mathcal{B}=\bigoplus_{i=0}^{\infty} B_{i} \subset \bigoplus_{i=0}^{\infty} A_{i l}$ be the subalgebra of $\mathcal{A}$ generated by $A_{l}$, and let $V_{d}=\operatorname{Span}\left\{a^{d} \mid a \in A_{l}\right\}, d \in \mathbb{N}$. Then $B_{d} \subseteq V_{d}$.

We relegate the proof to $\S 3.3$.

Theorem 3.1 may be restated as follows:

Corollary 3.5. Let $M^{2 n}$ be an $S^{1}$-manifold. Suppose, for some semisimple Lie group $G$, the $S^{1}$-action extends to a $G$-action such that every component of $M^{S^{1}}$ intersects $M^{G}$. Let $F_{0}$ be the union of those components of $M^{S^{1}}$ with codimensions congruent to $0 \bmod 4$, and let $i_{0}: F_{0} \hookrightarrow M$ be the inclusion map. Then $(\hat{\mathbf{L}}(M) \cdot C)[M]$ is determined by the submanifold $i_{0}: F_{0} \hookrightarrow M$ (and is independent of the $S^{1}$-action around $F_{0}$.) In particular, if, in addition to the stated condition, $F_{0}=\varnothing$, then $(\hat{\mathbf{L}}(M) \cdot C)[M]=0$.

3.2. $(P \cdot C)[M]$. We now consider rational numbers of the form $(P \cdot C)\left[M^{2 n}\right]$. The main results of this subsection are Theorems 3.6 and 3.11 .

Theorem 3.6. Let $G$ be semisimple and let $M^{2 n}$ be a $G$-manifold. Suppose there exists a circle subgroup $S \subset G$ such that every component of $M^{S}$ intersects $M^{G}$. Then $(P \cdot C)[M]$ can be determined in terms of the submanifold $i: M^{S} \hookrightarrow M$ and the $S$-action on the normal bundle $N$ of $M^{S} \hookrightarrow M$. To be precise, this number can be computed in terms of $i^{*}(C), p\left(M^{S}\right)$ (the total Pontrjagin class of $\left.M^{S}\right),\left[M^{S}\right]$, and, the action of $S$ on $N$.

The corresponding vanishing theorem is much simpler to state:

Corollary 3.7. Let $G$ be semisimple and let $M^{2 n}$ be a $G$-manifold. If, for some $g \in G, M^{g}=\varnothing$, then $(P \cdot C)[M]=0$.

Proof. If $M^{g}=\varnothing$, then $M^{T}=\varnothing$, where $T$ is some maximal torus containing $g$. As a standard fact about torus-actions, there always exists a circle subgroup $S \subset T$ such that $M^{S}=M^{T}$. Using such an $S$ and applying Theorem 3.6 yields the desired result.

In Corollary 4.9, this result will be considerably strengthened.

We now prepare for the proof of Theorem 3.6.

As before, endow $M^{2 n}$ with a Riemannian structure so that $G$ acts isometrically. Let $h: M^{2 n} \rightarrow B S O(2 n)$ be the classifying map for the tangent bundle $T M$. Define

$$
\mathfrak{b}: R S O(2 n) \longrightarrow K(B S O(2 n))
$$

by

$$
\mathfrak{b}: V \mapsto E S O(2 n) \times_{S O(2 n)} V
$$


$\mathfrak{b}$ "bundlizes" a complex $S O(2 n)$-module. On elements of $K(M)$ associated with the Riemannian structure, i.e., on elements in $\left(h^{*} \circ \mathfrak{b}\right)(R S O(2 n))$, the Chern character admits a universal interpretation as a ring homomorphism $\mathfrak{C h}$ on the complex $S O(2 n)$-representations to the cohomology of $B S O(2 n)$ :

$$
\mathfrak{C h}: R S O(2 n) \stackrel{\mathfrak{b}}{\longrightarrow} K(B S O(2 n)) \stackrel{\mathrm{ch}}{\longrightarrow} H^{*}(B S O(2 n) ; \mathbb{Q}) .
$$

Let $\sigma_{d}$ be the elementary symmetric polynomial of degree $d$ in $n$ variables. Let $x_{1}, \cdots, x_{n}$ be the basic characters of the maximal torus $T$ of $S O(2 n)$, regarded as cohomology classes in $H^{2}(B T ; \mathbb{Q})$. As usual, we identify $H^{*}(B S O(2 n) ; \mathbb{Q})$ with $H^{*}(B T ; \mathbb{Q})^{W}$, the subring of $H^{*}(B T ; \mathbb{Q})$ invariant under the Weyl group $W$.

Lemma $3.8([2])$. There exists $U_{\sigma_{d}} \in R S O(2 n)$ such that

$$
\mathfrak{C h}\left(U_{\sigma_{d}}\right)=\sigma_{d}\left(x_{1}^{2}, \cdots, x_{n}^{2}\right)+\text { higher dimensional terms. }
$$

Those higher dimensional terms do not involve the Euler class $\prod_{i=1}^{n} x_{i}$.

We now use this result to immediately deduce:

Lemma 3.9. Let $P_{d}\left(p_{1}, \cdots, p_{d}\right) \in H^{4 d}\left(M^{2 n}\right)$ be a homogeneous 4d-dimensional integral polynomial in the first d Pontrjagin classes $p_{1}, \cdots, p_{d}$ of a $G$-manifold $M^{2 n}$. Then there exists $E\left(P_{d}\right) \in K_{G}(M)$ with $\operatorname{ch} E\left(P_{d}\right)=P_{d}\left(p_{1}, \cdots, p_{d}\right)$.

Proof. Let $r=\left[\frac{n}{2}\right]$. We apply a finite induction on $d$.

First we consider the case where $d=r$. Let $\tilde{U}_{\sigma_{i}}=\mathfrak{b}\left(U_{\sigma_{i}}\right) \in K(B S O(2 n))$, and let

$$
E\left(P_{r}\right)=h^{*}\left(P_{r}\left(\tilde{U}_{\sigma_{1}}, \cdots, \tilde{U}_{\sigma_{r}}\right)\right) \in K(M) .
$$

Note that, because $h$ classifies the principal $S O(2 n)$-bundle, $\tau$, associated with the Riemannian structure of $M, E\left(P_{r}\right)=\tau \times_{S O(2 n)} P_{r}\left(U_{\sigma_{1}}, \cdots, U_{\sigma_{r}}\right)$. Hence, $E\left(P_{r}\right)$ admits a $G$-action induced by the $G$-action on $\tau$, i.e., $E\left(P_{r}\right) \in K_{G}(M)$. Then

$$
\begin{aligned}
\operatorname{ch} E\left(P_{r}\right) & =\operatorname{ch} h^{*}\left(P_{r}\left(\tilde{U}_{\sigma_{1}}, \cdots, \tilde{U}_{\sigma_{r}}\right)\right) \\
& =h^{*} \operatorname{ch} \mathfrak{b}\left(P_{r}\left(U_{\sigma_{1}}, \cdots, U_{\sigma_{r}}\right)\right) \\
& =h^{*} \mathfrak{C h}\left(P_{r}\left(U_{\sigma_{1}}, \cdots, U_{\sigma_{r}}\right)\right) \\
& =h^{*} P_{r}\left(\mathfrak{C h} U_{\sigma_{1}}, \cdots, \mathfrak{C h} U_{\sigma_{r}}\right) \\
& =h^{*}\left(P_{r}\left(\sigma_{1}, \cdots, \sigma_{r}\right)+\text { higher dimensional terms }\right) \\
& =P_{r}\left(h^{*} \sigma_{1}, \cdots, h^{*} \sigma_{r}\right)+\text { higher dimensional terms } \\
& =P_{r}\left(p_{1}, \cdots, p_{r}\right)+\text { higher dimensional terms } \\
& =P_{r}\left(p_{1}, \cdots, p_{r}\right)
\end{aligned}
$$

where the "higher dimensional terms" are of dimension at least $4(r+1)>2 n$ and hence are 0 .

Next we examine the case where $d=r-1$. Let

$$
E^{\prime}\left(P_{r-1}\right)=h^{*}\left(P_{r-1}\left(\tilde{U}_{\sigma_{1}}, \cdots, \tilde{U}_{\sigma_{r-1}}\right)\right) \in K(M) .
$$

Then

$$
\begin{aligned}
\operatorname{ch} E^{\prime}\left(P_{r-1}\right) & =P_{r-1}\left(p_{1}, \cdots, p_{r-1}\right)+\text { higher dimensional terms } \\
& =P_{r-1}\left(p_{1}, \cdots, p_{r-1}\right)+Q_{r}\left(p_{1}, \cdots, p_{r}\right)
\end{aligned}
$$


where $Q_{r}\left(p_{1}, \cdots, p_{r}\right)$ is a homogeneous $4 r$-dimensional integral polynomial. Simply let $E\left(P_{r-1}\right)=E^{\prime}\left(P_{r-1}\right)-E\left(Q_{r}\right)$; then

$$
\begin{aligned}
\operatorname{ch} E\left(P_{r-1}\right) & =\operatorname{ch}\left(E^{\prime}\left(P_{r-1}\right)-E\left(Q_{r}\right)\right) \\
& =\operatorname{ch} E^{\prime}\left(P_{r-1}\right)-\operatorname{ch} E\left(Q_{r}\right) \\
& =P_{r-1}\left(p_{1}, \cdots, p_{r-1}\right) .
\end{aligned}
$$

Continuing in this fashion until reaching the case where $d=0$ proves the result.

Since $\operatorname{Sign}_{G}(M ; E)$ is additive in $E, E$ can be allowed to be an element of $K_{G}(M)$.

Proof of Theorem 3.6. By Remark 3.2, we assume that $G$ is simply connected and $G$ acts isometrically on $M$. The $G$-action preserves the orientation of $M$, as $G$ is connected.

By Lemma 3.4, it suffices to prove the result for $\left\{c^{n-2 d} \cdot P_{d}\right\}\left[M^{2 n}\right]$ where $c \in \operatorname{Im}\left(H^{2}(M) \rightarrow H^{2}(M ; \mathbb{Q})\right)$ and $P_{d} \in H^{4 d}\left(M^{2 n} ; \mathbb{Q}\right)$ is a homogeneous $4 d$ dimensional polynomial in the Pontrjagin classes of $M^{2 n}$.

Let $\mathcal{L}(c) \rightarrow M$ be the complex line bundle whose first Chern class is $c$. Following the same reasoning as in the proof of Theorem 3.1, the $G$-action on $M$ admits a (unique) lifting to a $G$-action on $\mathcal{L}(c)$ and $S$ acts trivially on $\mathcal{L}(c)_{x}$ for every $x \in M_{\nu}^{S}$.

Construct $E\left(P_{d}\right)$ as in Lemma 3.9. We consider $\operatorname{Sign}_{G}\left(M ; \mathcal{L}(c) \otimes E\left(P_{d}\right)\right)(z)$ for $z \in S$. We use the same notation as in the proof of Theorem 3.1. Let $z$ be (the complex $S^{1}$-coordinate of) any topological generator of $S$. Then $M^{z}=M^{S}$. Calculating just as in the proof of Theorem 3.1, we have

$$
\begin{aligned}
& \operatorname{Sign}_{G}\left(M ; \mathcal{L}(c) \otimes E\left(P_{d}\right)\right)(z) \\
& =\operatorname{Sign}_{S}\left(M ; \mathcal{L}(c) \otimes E\left(P_{d}\right)\right)(z) \\
& =\sum_{\nu} 2^{t_{\nu}} \operatorname{ch}\left(\left.\mathcal{L}(c) \otimes E\left(P_{d}\right)\right|_{M_{\nu}^{S}}\right)(z) \hat{\mathbf{L}}\left(M_{\nu}^{S}\right) \prod_{k \in \mathbb{N}} \prod_{j=1}^{d_{\nu}(k)} \frac{z^{k}+e^{-x_{\nu, j}(k)}}{z^{k}-e^{-x_{\nu, j}(k)}}\left[M_{\nu}^{S}\right] \\
& =\sum_{\nu} 2^{t_{\nu}} e^{i_{\nu}^{*}(c)} z^{l_{\nu}} \operatorname{ch}\left(\left.E\left(P_{k}\right)\right|_{M_{\nu}^{S}}\right)(z) \hat{\mathbf{L}}\left(M_{\nu}^{S}\right) \prod_{k \in \mathbb{N}} \prod_{j=1}^{d_{\nu}(k)} \frac{z^{k}+e^{-x_{\nu, j}(k)}}{z^{k}-e^{-x_{\nu, j}(k)}}\left[M_{\nu}^{S}\right] \\
& =\sum_{\nu} 2^{t_{\nu}} e^{i_{\nu}^{*}(c)} \operatorname{ch}\left(\left.E\left(P_{k}\right)\right|_{M_{\nu}^{S}}\right)(z) \hat{\mathbf{L}}\left(M_{\nu}^{S}\right) \prod_{k \in \mathbb{N}} \prod_{j=1}^{d_{\nu}(k)} \frac{z^{k}+e^{-x_{\nu, j}(k)}}{z^{k}-e^{-x_{\nu, j}(k)}}\left[M_{\nu}^{S}\right] .
\end{aligned}
$$

The complex cohomology class

$$
\operatorname{ch}\left(\left.E\left(P_{d}\right)\right|_{M_{\nu}^{S}}\right)(z)
$$

by definition is determined by the submanifold $M_{\nu}^{S} \stackrel{i_{\nu}}{\longrightarrow} M$ and the $S$-action around it. Hence the above formula shows that the complex number

$$
\operatorname{Sign}_{G}\left(M ; \mathcal{L}(c) \otimes E\left(P_{d}\right)\right)(z)
$$


is determined by the data of the submanifold $i: M^{S} \hookrightarrow M$ and the $S$-action on $N$. By continuity of index as a character function on $G$, the integer

$$
\begin{aligned}
\operatorname{Sign}\left(M ; \mathcal{L}(c) \otimes E\left(P_{d}\right)\right) & =\operatorname{Sign}_{G}\left(M ; \mathcal{L}(c) \otimes E\left(P_{d}\right)\right)(e) \\
& =\lim _{z \rightarrow 1} \operatorname{Sign}_{S}\left(M ; \mathcal{L}(c) \otimes E\left(P_{d}\right)\right)(z)
\end{aligned}
$$

is also determined by the data of the submanifold $i: M^{S} \hookrightarrow M$ and the $S$-action on $N$. Let $2 n=4 r$ or $4 r+2$. An explicit formula for this limit when $d=r$ is to be given in Remark 3.10; when $d<r$, the limit can be calculated using an induction shown below.

Using Theorem 2.7, we have

$$
\begin{aligned}
& \operatorname{Sign}\left(M ; \mathcal{L}(c) \otimes E\left(P_{d}\right)\right) \\
& =2^{n}\left\{\operatorname{ch}\left(\mathcal{L}(c) \otimes E\left(P_{d}\right)\right) \cdot \hat{\mathbf{L}}(M)\right\}[M] \\
& =2^{n}\left\{e^{c} \cdot P_{d} \cdot \hat{\mathbf{L}}(M)\right\}[M] .
\end{aligned}
$$

We apply a finite induction on $d$. First we examine the case where $d=r$. Then,

$$
\begin{aligned}
& \operatorname{Sign}\left(M ; \mathcal{L}(c) \otimes E\left(P_{r}\right)\right) \\
& =2^{n}\left\{e^{c} \cdot P_{r} \cdot \hat{\mathbf{L}}(M)\right\}\left[M^{2 n}\right] \\
& = \begin{cases}2^{2 r} P_{r}\left[M^{4 r}\right] & \text { when } 2 n=4 r, \\
2^{2 r+1}\left\{c \cdot P_{r}\right\}\left[M^{4 r+2}\right] & \text { when } 2 n=4 r+2,\end{cases}
\end{aligned}
$$

proving the result for $d=r$.

Next we examine the case where $d=r-1$. Then

$$
\begin{aligned}
& \operatorname{Sign}\left(M ; \mathcal{L}(c) \otimes E\left(P_{r-1}\right)\right) \\
& =2^{n}\left\{\left(\sum_{j=0}^{n} \frac{c^{j}}{j !}\right) \cdot P_{r-1} \cdot\left(\sum_{i=0}^{r} \hat{L}_{i}(M)\right)\right\}\left[M^{2 n}\right] \\
& =\left\{\begin{array}{l}
2^{2 r}\left\{\frac{c^{2}}{2 !} \cdot P_{r-1}+P_{r-1} \cdot \hat{L}_{1}\right\}\left[M^{4 r}\right] \\
2^{2 r+1}\left\{\frac{c^{3}}{3 !} \cdot P_{r-1}+c \cdot P_{r-1} \cdot \hat{L}_{1}\right\}\left[M^{4 r+2}\right]
\end{array} \text { when } 2 n=4 r,\right. \\
&
\end{aligned}
$$

Note that $P_{r-1} \cdot \hat{L}_{1}(M)$ is a homogeneous $4 r$-dimensional polynomial in the Pontrjagin classes, and thus, by the proven case (in which $d=r$ ),

$$
\left\{P_{r-1} \cdot \hat{L}_{1}\left(M^{4 r}\right)\right\}\left[M^{4 r}\right]
$$

and

$$
\left\{c \cdot P_{r-1} \cdot \hat{L}_{1}\left(M^{4 r+2}\right)\right\}\left[M^{4 r+2}\right]
$$

can be calculated by localization. Hence, so can

$$
\left\{c^{2} \cdot P_{r-1}\right\}\left[M^{4 r}\right]
$$

and

be calculated by localization.

$$
\left\{c^{3} \cdot P_{r-1}\right\}\left[M^{4 r+2}\right]
$$

Continuing in this fashion until reaching the case where $d=0$ completes the induction. 
Remark 3.10. We now indicate how to calculate $P_{r}\left[M^{4 r}\right]$ or $\left(c \cdot P_{r}\right)\left[M^{4 r+2}\right]$ using fixed-point data, which is our first inductive step in the proof of Theorem 3.6.

Observe that when $\operatorname{dim} M=4 r$, the localization result for $P_{r}[M]$ requires neither the semisimplicity of $G$ nor the relation between $M^{S^{1}}$ and $M^{G}$ because $\mathcal{L}(c)$ does not figure in the calculation. For this case, an explicit localization formula is given in [2]. We first briefly describe this formula; we then indicate one for $\left(c \cdot P_{r}\right)[M]$ when $\operatorname{dim} M=4 r+2$.

In light of the splitting principle, $P_{r}\left(p_{1}, \cdots, p_{r}\right)$ may be formally regarded as a polynomial $P_{r}^{\prime}\left(x_{1}^{2}, \cdots, x_{n}^{2}\right)$ symmetric in $x_{1}^{2}, \cdots, x_{n}^{2}$ (where $x_{1}, \cdots, x_{n}$ can be regarded as the basic characters of the maximal torus $T$ of $S O(2 n))$. We will write the formulae in terms of $P_{r}^{\prime}$.

First let $\operatorname{dim} M=2 n=4 r . S^{1}$ acts on the normal bundle $N_{\nu}$ with weights (counting multiplicity) $\omega_{\nu, j} \in \mathbb{Z}, j=1, \cdots, n-t_{\nu}$; let $y_{\nu, j}$ be the corresponding Chern roots. (In detail, one constructs a "flag bundle" $P_{\nu} \stackrel{\pi}{\rightarrow} M_{\nu}^{S^{1}}$ such that $\pi^{*} N_{\nu}=\bigoplus_{j} \eta_{\nu, j}$, each $\eta_{\nu, j}$ being an $S^{1}$-invariant complex line bundle on which $z \in S^{1}$ acts via multiplication by $z^{\omega_{\nu, j}}$; and $y_{\nu, j}=c_{1}\left(\eta_{\nu, j}\right)$.)

Using index theory, [2] shows that, formally,

$$
\begin{aligned}
& P_{r}\left(p_{1}, \cdots, p_{r}\right)\left[M^{4 r}\right] \\
& =\frac{1}{2^{n}} \lim _{z \rightarrow 1} \operatorname{Sign}_{S^{1}}\left(M ; E\left(P_{r}\right)\right)(z) \\
& =\sum_{\nu} \frac{P_{r}^{\prime}\left(x_{1}^{\prime 2}, \cdots, x_{t_{\nu}}^{\prime 2} ;\left(y_{\nu, 1}+i \omega_{\nu, 1}\right)^{2}, \cdots,\left(y_{\nu, n-t_{v}}+i \omega_{\nu, n-t_{v}}\right)^{2}\right)}{\prod_{j=1}^{n-t_{v}}\left(y_{\nu, j}+i \omega_{\nu, j}\right)}\left[M_{\nu}^{S^{1}}\right]
\end{aligned}
$$

where $x_{1}^{\prime 2}, \cdots, x_{t_{\nu}}^{\prime 2}$ are the formal "Pontrjagin roots" of $M_{\nu}^{S^{1}}$.

Using a calculation identical to the one which leads 2 to the above formula, one easily obtains, when $\operatorname{dim} M=2 n=4 r+2$, that

$$
\begin{aligned}
& \left\{c \cdot P_{r}\left(p_{1}, \cdots, p_{r}\right)\right\}\left[M^{4 r+2}\right] \\
& =\frac{1}{2^{n}} \lim _{z \rightarrow 1} \operatorname{Sign}_{S^{1}}\left(M ; \mathcal{L}(c) \otimes E\left(P_{r}\right)\right)(z) \\
& =\sum_{\nu} \frac{c \cdot P_{r}^{\prime}\left(x_{1}^{\prime 2}, \cdots, x_{t_{\nu}}^{\prime 2} ;\left(y_{\nu, 1}+i \omega_{\nu, 1}\right)^{2}, \cdots,\left(y_{\nu, n-t_{v}}+i \omega_{\nu, n-t_{v}}\right)^{2}\right)}{\prod_{j=1}^{n-t_{v}}\left(y_{\nu, j}+i \omega_{\nu, j}\right)}\left[M_{\nu}^{S^{1}}\right] .
\end{aligned}
$$

Using these formulae and the induction steps shown in the proof of Theorem 3.6, one can then calculate $\left\{c^{n-2 d} \cdot P_{d}\left(p_{1}, \cdots, p_{d}\right)\right\}\left[M^{2 n}\right]$ by localization.

In (the proofs of) Theorem 3.1 and 3.6, the determination of certain "characteristic numbers" involving $c$ is seen to require no knowledge of the lifted $G$-action on $\mathcal{L}(c)$; this relies on the fact that the $G$-action on $\left.\mathcal{L}(c)\right|_{M^{S}}$ is trivial, a consequence of the semisimplicity of $G$ and the condition on the relation between $M^{S}$ and $M^{G}$.

In general, when $M^{S}=\varnothing$, to deduce the vanishing of the numbers in question, we only need to know the existence of a lifting of the $G$-action to an action on $\mathcal{L}(c)$ without having to know how to produce one; Corollaries 2.2 and 2.3 give some sufficient conditions for the existence of a lifting. When $M^{S} \neq \varnothing$, a "localization" result on the numbers in question will require the knowledge of a lifting. We summarize this discussion in the following theorem. 
Theorem 3.11. Given a $G$-manifold $M$, let $c_{j} \in \operatorname{Im}\left(H^{2}(M) \rightarrow H^{2}(M ; \mathbb{Q})\right)$ and $R=\left(P \cdot \prod_{j} c_{j}\right)[M]$.

If either $\pi_{1}(G)=\{0\}$ or $H^{1}(M)=\{0\}$, then, given any circle subgroup $S \subset G$, $R$ can be determined in terms of: the submanifold $M^{S} \hookrightarrow M$, the $S$-action around $M^{S}$, and the restriction to $\left.\mathcal{L}\left(c_{j}\right)\right|_{M^{S}}$ of any lifted $S$-action on each $\mathcal{L}\left(c_{j}\right)$.

If $G$ is semisimple, then by regarding $M$ as a $\widetilde{G}$-manifold where $\widetilde{G}$ is the universal cover of $G, R$ can be similarly localized as in the previous case.

In either case, if $M^{g}=\varnothing$ for some $g \in G$, then $R=0$.

This is clear from the calculations we have done and Corollary 2.2.

We now use the last result to make an observation. [7 shows that if $X$ is a $T$ space ( $T$ being a torus) with vanishing even-dimensional rational homotopy groups, then $X^{T}$ is either empty or connected. Proposition 3.3 shows that if $M$ is an evendimensional manifold admitting a nontrivial $T$-action, then $M^{T}$ is either empty or disconnected. Hence,

Corollary 3.12. Let $M$ be an even-dimensional manifold satisfying $H^{1}(M)=\{0\}$ and $\pi_{2 k}(M) \otimes \mathbb{Q}=\{0\}$ for all $k \in \mathbb{N}$. If, for some classes $c_{j} \in H^{2}(M ; \mathbb{Q})$ and some rational polynomial $P$ in the Pontrjagin classes of $M,\left(P \cdot \prod_{j} c_{j}\right)[M] \neq 0$, then $M$ admits no nontrivial torus-action.

Proof. Suppose $M$ admits a $T$-action, $T$ being some torus. As $H^{1}(M)=\{0\}$ and $\left(P \cdot \prod_{j} c_{j}\right)[M] \neq 0, M^{T} \neq \varnothing$ by Theorem 3.11. As $\pi_{2 k}(M) \otimes \mathbb{Q}=\{0\}, M^{T}$ must be connected by the result of 7 quoted above; as $\operatorname{dim} M$ is even, $M^{T}$ must be disconnected by Proposition 3.3. This contradiction proves the result.

This implies a result of [12, which shows that, when $M$ is as above, the nonvanishing of $\left(\prod_{j} c_{j}\right)[M]$ is an obstruction to $S^{1}$-actions on $M$. An example is yet to be seen in which Corollary 3.12 is genuinely stronger.

3.3. Proof of Lemma 3.4. We now prove Lemma 3.4.

Lemma 3.13. Suppose $\mathcal{A}=\bigoplus_{i=0}^{\infty} A_{i}$ is a graded $\mathbb{k}$-algebra, $\mathbb{k}$ being a field of characteristic 0 . Let $l$ be a given positive integer, let $\mathcal{B}=\bigoplus_{i=0}^{\infty} B_{i} \subset \bigoplus_{i=0}^{\infty} A_{i l}$ be the subalgebra of $\mathcal{A}$ generated by $A_{l}$, and let $V_{d}=\operatorname{Span}\left\{a^{d} \mid a \in A_{l}\right\}, d \in \mathbb{N}$. Then $B_{d} \subseteq V_{d}$.

Proof. What needs to be shown is the fact that an element in $B_{d}$ of the form

$$
\prod_{j=1}^{m} c_{j}^{d_{j}}, \text { with } c_{j} \in A_{l}, d_{j}>0, \text { and } \sum_{j=1}^{m} d_{j}=d
$$

can be written as a linear combination of elements of the form $a^{d}$ with $a \in A_{l}$.

We apply induction on the number $m$ of distinct $A_{l}$-factors in (3.5). The case of $m=1$ is automatic. Assuming the result for all $m<N$, it is then to be shown that it holds for $m=N$.

In what follows, we adopt the multi-index notation: $\xi=\left(\xi_{1}, \cdots, \xi_{N}\right)$ where $\xi_{j}$ are nonnegative integers, $|\xi|=\sum_{j=1}^{N} \xi_{j}, \mathbf{c}=\left(c_{1}, \cdots, c_{N}\right)$ where $c_{j} \in A_{l}, \mathbf{c}^{\xi}=\prod_{j=1}^{N} c_{j}^{\xi_{j}}$, $M_{\xi}$ are the multinomial coefficients. 
Let $S(\mathbf{c})=\left(c_{1}+\cdots+c_{N}\right)^{d}$. Consider the multinomial expansion of $S(\mathbf{c})$ :

$$
S(\mathbf{c})=S_{0}(\mathbf{c})+T(\mathbf{c})
$$

where

$$
S_{0}(\mathbf{c})=\sum_{|\xi|=d, \text { and }} M_{\xi} \cdot \mathbf{c}^{\xi}
$$

and

$$
T(\mathbf{c})=\sum_{|\xi|=d, \text { and } \xi_{j}>0 \text { for all } j} M_{\xi} \cdot \mathbf{c}^{\xi} .
$$

Our task is to show that each summand in $T(\mathbf{c})$ can be written as a linear combination of elements of the form $a^{d}$ with $a \in A_{l}$.

In $S_{0}(\mathbf{c})$, each summand contains fewer than $N$ distinct $A_{l}$-factors; thus, by the induction hypothesis, $S_{0}(\mathbf{c}) \in V_{d}$. Since $T(\mathbf{c})=\left(\sum c_{j}\right)^{d}-S_{0}(\mathbf{c}), T(\mathbf{c}) \in V_{d}$. We now examine $T(\mathbf{c})$ more closely:

$$
\begin{aligned}
T(\mathbf{c}) & =\sum_{\xi_{1}=1}^{d-(N-1)}\left(c_{1}^{\xi_{1}} \cdot \sum_{\substack{\xi_{2}+\cdots+\xi_{N}=d-\xi_{1} \\
\xi_{j}>0}}\left(M_{\xi} \prod_{j=2}^{N} c_{j}^{\xi_{j}}\right)\right) \\
& =\sum_{\xi_{1}=1}^{d-(N-1)}\left\{c_{1}^{\xi_{1}} \cdot \sum_{\xi_{2}=1}^{d-(N-2)-\xi_{1}}\left(c_{2}^{\xi_{2}} \cdot \sum_{\substack{\xi_{3}+\cdots+\xi_{N}=d-\left(\xi_{1}+\xi_{2}\right) \\
\xi_{j}>0}}\left(M_{\xi} \prod_{j=3}^{N} c_{j}^{\xi_{j}}\right)\right)\right\} \\
& =\cdots .
\end{aligned}
$$

For each $i \in\{1, \cdots,(N-1)\}$, let

$$
T\left(\mathbf{c} ; \xi_{1}, \cdots, \xi_{i}\right)=\sum_{\substack{\xi_{i+1}+\cdots+\xi_{N}=d-\left(\xi_{1}+\cdots+\xi_{i}\right) \\ \xi_{j}>0}}\left(M_{\xi} \prod_{j=i+1}^{N} c_{j}^{\xi_{j}}\right) .
$$

We then can rewrite the above as:

$$
\begin{aligned}
T(\mathbf{c}) & =\sum_{\xi_{1}=1}^{d-(N-1)}\left(c_{1}^{\xi_{1}} T\left(\mathbf{c} ; \xi_{1}\right)\right) \\
& =\sum_{\xi_{1}=1}^{d-(N-1)}\left\{c_{1}^{\xi_{1}} \cdot \sum_{\xi_{2}=1}^{d-(N-2)-\xi_{1}}\left(c_{2}^{\xi_{2}} T\left(\mathbf{c} ; \xi_{1}, \xi_{2}\right)\right)\right\} \\
& =\cdots .
\end{aligned}
$$

Let $\mathbf{c}_{(i, t)}=\left(c_{1}, \cdots, t c_{i}, \cdots, c_{N}\right)$ be the $N$-tuple obtained from $\mathbf{c}$ by replacing the $i$ th entry $c_{i}$ with $t c_{i}$. Consider the system of linear equations in $\left(c_{1}^{\xi_{1}} T\left(\mathbf{c} ; \xi_{1}\right)\right)$, $1 \leq \xi_{1} \leq d-(N-1)$ :

$$
\sum_{\xi_{1}=1}^{d-(N-1)} t^{\xi_{1}}\left(c_{1}^{\xi_{1}} T\left(\mathbf{c} ; \xi_{1}\right)\right)=T\left(\mathbf{c}_{(1, t)}\right), \quad t=1, \cdots, d-(N-1) .
$$


The coefficients of this linear system form a Vandermonde matrix, hence this system is invertible. As $T\left(\mathbf{c}_{(1, t)}\right) \in V_{d}$ for each $t$, we deduce that

$$
\left(c_{1}^{\xi_{1}} T\left(\mathbf{c} ; \xi_{1}\right)\right) \in V_{d}, \quad \text { for } 1 \leq \xi_{1} \leq d-(N-1) .
$$

By (3.6), for each $\xi_{1}$,

$$
\begin{aligned}
c_{1}^{\xi_{1}} T\left(\mathbf{c} ; \xi_{1}\right) & =c_{1}^{\xi_{1}} \cdot \sum_{\xi_{2}=1}^{d-(N-2)-\xi_{1}}\left(c_{2}^{\xi_{2}} \cdot T\left(\mathbf{c} ; \xi_{1}, \xi_{2}\right)\right) \\
& =\sum_{\xi_{2}=1}^{d-(N-2)-\xi_{1}}\left(c_{1}^{\xi_{1}} c_{2}^{\xi_{2}} T\left(\mathbf{c} ; \xi_{1}, \xi_{2}\right)\right) .
\end{aligned}
$$

Given $\xi_{1}$, consider the following linear system in $c_{1}^{\xi_{1}} c_{2}^{\xi_{2}} T\left(\mathbf{c} ; \xi_{1}, \xi_{2}\right)$ :

$$
\sum_{\xi_{2}=1}^{d-(N-2)-\xi_{1}} t^{\xi_{2}} c_{1}^{\xi_{1}} c_{2}^{\xi_{2}} T\left(\mathbf{c} ; \xi_{1}, \xi_{2}\right)=c_{1}^{\xi_{1}} T\left(\mathbf{c}_{(2, t)} ; \xi_{1}\right), t=1, \cdots, d-(N-2)-\xi_{1} .
$$

The coefficients of this linear system form a Vandermonde matrix, hence this system is invertible. By $(3.7), c_{1}^{\xi_{1}} T\left(\mathbf{c}_{(2, t)} ; \xi_{1}\right) \in V_{d}$ for each $t$, from which we deduce that

$$
c_{1}^{\xi_{1}} c_{2}^{\xi_{2}} T\left(\mathbf{c} ; \xi_{1}, \xi_{2}\right) \in V_{d} \text {, for } 1 \leq \xi_{1} \leq d-(N-1) \text { and } 1 \leq \xi_{2} \leq d-(N-2)-\xi_{1} \text {. }
$$

Continuing this argument, we would arrive finally at

$$
\left(\prod_{j=1}^{N-1} c_{j}^{\xi_{j}}\right) \cdot T\left(\mathbf{c} ; \xi_{1}, \cdots, \xi_{N-1}\right) \in V_{d}
$$

By definition,

$$
\begin{aligned}
T\left(\mathbf{c} ; \xi_{1}, \cdots, \xi_{N-1}\right) & =\sum_{\substack{\xi_{N}=d-\left(\xi_{1}+\cdots+\xi_{N-1}\right) \\
\xi_{j}>0}}\left(M_{\xi} \prod_{j=N}^{N} c_{j}^{\xi_{j}}\right) \\
& =M_{\xi} c_{N}^{d-\left(\xi_{1}+\cdots+\xi_{N-1}\right)} .
\end{aligned}
$$

Hence, for $|\xi|=d$,

$$
\left(\prod_{j=1}^{N} c_{j}^{\xi_{j}}\right) \in V_{d}
$$

as desired.

\section{ObstruCtions From the FUndamental Group}

In this section, we study obstructions, stemming from $\pi_{1}(M)$, to the existence of certain group actions on $M$. The results produced here are "higher" versions of those in $\S 3$ in the following sense.

Given a manifold $M$, an element $x \in H^{*}(\pi ; \mathbb{Q})$ determines a rational characteristic number $\left(f^{*}(x) \cdot \hat{\mathbf{L}}(M)\right)[M]$, which S. P. Novikov calls "a higher signature of $M$ ". 3] calls the number $\left(f^{*}(x) \cdot \hat{\mathbf{A}}(M)\right)[M]$ "a higher $\hat{\mathbf{A}}$-genus of $M$ ".

In this section, we consider, for certain $x \in H^{*}(\pi ; \mathbb{Q})$, numbers of the form $\left(f^{*}(x) \cdot P \cdot C\right)[M]$. For example, we show, in Theorem 4.8, that if $M$ admits an 
action by a semisimple Lie group such that some group element acts without fixed point, then $\left(f^{*}(x) \cdot P \cdot C\right)[M]=0$.

We first review, in $\S 4.1$, techniques developed by [3], of which we note some immediate consequences. We then state and prove some "higher" vanishing theorems in $\S 4.2$.

4.1. $G$-actions and the fundamental group. In $\S 4.1 .1$, we introduce [3]'s work relating the fundamental group of a $G$-manifold to the study of the $G$-action and we derive some direct consequences of it. In $\S 4.1 .2$, we note an application.

4.1.1. Relations among $H^{*}(M), H^{*}(M / G)$, and $\pi_{1}(M)$. Suppose $G$ acts effectively on $M$. Let $\Gamma$ be a (discrete) group and $s: M \rightarrow K(\Gamma, 1)$ a continuous map inducing a surjective homomorphism $s_{*}: \pi \rightarrow \Gamma$. Let $q: M \rightarrow M / G$ be the canonical quotient map and $o: G \rightarrow M$ the orbit of some basepoint of $M$. It is a standard fact that $o_{*}\left(\pi_{1}(G)\right) \subseteq \mathcal{Z}(\pi)$ (the center of $\pi$ ); from this and the surjectivity of $s_{*}$, it follows that $s_{*} o_{*}\left(\pi_{1}(G)\right) \subseteq \mathcal{Z}(\Gamma)$. Let $\Gamma_{0}=\Gamma / s_{*} o_{*}\left(\pi_{1}(G)\right)$ and let $\rho: \Gamma \rightarrow \Gamma_{0}$ be the quotient map.

Lemma 4.1 ([3]). Let $G, M, \Gamma, \Gamma_{0}, s, \rho$, and $q$ be as above. There exists a map

$$
\varphi: H^{*}\left(\Gamma_{0} ; \mathbb{Q}\right) \longrightarrow H^{*}(M / G ; \mathbb{Q})
$$

making the following diagram commute:

$$
\begin{array}{cccc}
H^{*}\left(\Gamma_{0} ; \mathbb{Q}\right) & \stackrel{\varphi}{\longrightarrow} & H^{*}(M / G ; \mathbb{Q}) \\
\downarrow \rho^{*} & & \downarrow q^{*} \\
H^{*}(\Gamma ; \mathbb{Q}) & \stackrel{s^{*}}{\longrightarrow} & H^{*}(M ; \mathbb{Q})
\end{array}
$$

$\varphi$ is not necessarily induced by a map between spaces. Taking duals of the maps yields a similar commutative diagram of homology groups.

We note some consequences of this lemma.

If $G$ is semisimple, or $\pi$ has finite center, or $M^{G} \neq \varnothing$, then $s_{*} o_{*}\left(\pi_{1}(G)\right)$ is finite, in which case

is an isomorphism.

$$
\rho^{*}: H^{*}\left(\Gamma_{0} ; \mathbb{Q}\right) \longrightarrow H^{*}(\Gamma ; \mathbb{Q})
$$

Another sufficient condition for the finiteness of $s_{*} O_{*}\left(\pi_{1}(G)\right)$ is that the isotropy subgroup $G_{a} \stackrel{j}{\hookrightarrow} G$ be connected and $\pi_{1}(G) / j_{*} \pi_{1}\left(G_{a}\right)$ be finite. To see this, first note that the orbit map $o$ factors as follows:

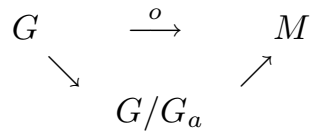

Note also that, for any connected subgroup $H \stackrel{j}{\hookrightarrow} G, \pi_{1}(G / H) \simeq \pi_{1}(G) / j_{*} \pi_{1}(H)$, which is easily seen via the long exact sequence of homotopy groups for the bundle $H \rightarrow G \rightarrow G / H$. Thus, under the stated condition, $o_{*}\left(\pi_{1}(G)\right)$ is finite.

We summarize this discussion in

Corollary 4.2. Let $G, M, \Gamma, o, s$, and $q$ be as in Lemma 4.1 except that $\pi \stackrel{s_{*}}{\rightarrow} \Gamma$ need not be a surjection. Suppose $o_{*}\left(\pi_{1}(G)\right)$ is finite, which is satisfied if any one of the following four conditions holds:

(1) $G$ is semisimple; 
(2) $\mathcal{Z}(\pi)$ is finite;

(3) $M^{G} \neq \varnothing$;

(4) there exists $a \in M$ such that its isotropy subgroup $G_{a} \stackrel{j}{\hookrightarrow} G$ is connected and $\pi_{1}(G) / j_{*} \pi_{1}\left(G_{a}\right)$ is finite.

Then there exists a map

$$
\varphi: H^{*}(\Gamma ; \mathbb{Q}) \longrightarrow H^{*}(M / G ; \mathbb{Q})
$$

making the following diagram commute:

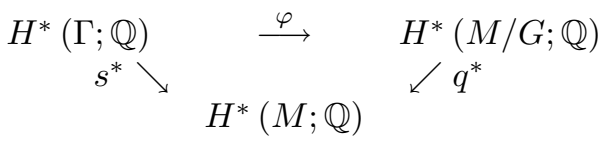

Proof. When $\pi \stackrel{s_{*}}{\rightarrow} \Gamma$ is a surjection, this result follows from Lemma 4.1 once $H^{*}(\Gamma ; \mathbb{Q})$ and $H^{*}\left(\Gamma_{0} ; \mathbb{Q}\right)$ are identified via the isomorphism $\rho^{*}$.

When $\pi \stackrel{s_{*}}{\rightarrow} \Gamma$ is not a surjection, $s_{*}$ can be canonically factored into $\pi \stackrel{s_{*}^{\prime}}{\rightarrow} \operatorname{Im} s_{*} \stackrel{i}{\hookrightarrow}$ $\Gamma$, a surjection followed by an inclusion. As $s_{*}^{\prime}$ is a surjection, we may apply the above result to obtain a map $\varphi^{\prime}$ making the following diagram commute:

$$
\begin{array}{rlrl}
H^{*}\left(\operatorname{Im} s_{*}^{\prime} ; \mathbb{Q}\right) & \stackrel{\varphi^{\prime}}{\longrightarrow} & H^{*}(M / G ; \mathbb{Q}) \\
\left(s^{\prime}\right)^{*} \searrow & & \swarrow q^{*} \\
& & H^{*}(M ; \mathbb{Q}) &
\end{array}
$$

We can then forge another commutative diagram:

$$
\begin{array}{ccc}
H^{*}(\Gamma ; \mathbb{Q}) & \stackrel{\varphi^{\prime} i^{*}}{\longrightarrow} & H^{*}(M / G ; \mathbb{Q}) \\
i^{*} \downarrow & & \downarrow q^{*} \\
H^{*}\left(\operatorname{Im} s^{*} ; \mathbb{Q}\right) & \stackrel{\left(s^{\prime}\right)^{*}}{\longrightarrow} & H^{*}(M ; \mathbb{Q})
\end{array}
$$

Letting $\varphi=\varphi^{\prime} i^{*}$, we have $q^{*} \varphi=\left(s^{\prime}\right)^{*} i^{*}=\left(i s^{\prime}\right)^{*}=s^{*}$, as desired.

We also mention that if $M \stackrel{f}{\rightarrow} K(\pi, 1)$ factors through $M / G$, then certainly a three-term diagram like the one in Corollary 4.2 (with $\Gamma=\pi$ and $s=f$ ) commutes. See [11] for a discussion of conditions that guarantee this.

Lemma 4.1 is stated under the assumption that the $G$-action on $M$ is effective. However, we can allow the action $G \rightarrow \operatorname{Diff}(M)$ to have a nontrivial but finite kernel. Such an action is said to be almost effective.

Corollary 4.3. If the G-action is almost effective, then the conclusion of Lemma 4.1 still holds.

Proof. For convenience, let $N=\operatorname{ker}(G \rightarrow \operatorname{Diff}(M))$ and $G^{\prime}=G / N$. Then $N \hookrightarrow$ $G \stackrel{p}{\rightarrow} G^{\prime}$ is a covering space of finite degree and hence $\pi_{1}\left(G^{\prime}\right) / p_{*} \pi_{1}(G)$ is finite. As we now have two actions on $M$, one being the almost effective $G$-action, and, the other the effective $G^{\prime}$-action, we let $\Gamma_{0}(G)$ and $\Gamma_{0}\left(G^{\prime}\right)$ denote respectively $\Gamma / s_{*} o_{*}\left(\pi_{1}(G)\right)$ and $\Gamma / s_{*} o_{*}^{\prime}\left(\pi_{1}\left(G^{\prime}\right)\right)$, where $o^{\prime}: G^{\prime} \rightarrow M$ is the $G^{\prime}$-orbit. Let $\rho^{\prime}: \Gamma \rightarrow \Gamma_{0}\left(G^{\prime}\right)$ be the quotient map, and let $h$ and $h^{\prime}$ abbreviate respectively $s_{*} O_{*}$ and $s_{*} o_{*}^{\prime}$. Note that $o=o^{\prime} p$; hence, $h=h^{\prime} p_{*}$. 
Since $G^{\prime}$ acts effectively on $M$ and $M / G=M / G^{\prime}$, there is a map $\varphi$ making the following diagram commute:

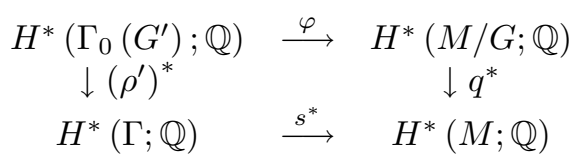

For our result, it suffices to prove that

$$
H^{*}\left(\Gamma_{0}(G) ; \mathbb{Q}\right) \simeq H^{*}\left(\Gamma_{0}\left(G^{\prime}\right) ; \mathbb{Q}\right) .
$$

We show this by showing that $\Gamma_{0}\left(G^{\prime}\right)$ is a quotient of $\Gamma_{0}(G)$ by a finite subgroup.

We first note that

$$
\Gamma_{0}\left(G^{\prime}\right) \simeq \frac{\Gamma_{0}(G)}{h^{\prime}\left(\pi_{1}\left(G^{\prime}\right)\right) / h\left(\pi_{1}(G)\right)} .
$$

This is simply a consequence of the definitions of the groups involved and the relevant isomorphism theorem in group theory. We next note that, since $p_{*} \pi_{1}(G)$ has finite index in $\pi_{1}\left(G^{\prime}\right), h^{\prime} p_{*}\left(\pi_{1}(G)\right)$ (which equals $h\left(\pi_{1}(G)\right)$ ) also has finite index in $h^{\prime}\left(\pi_{1}\left(G^{\prime}\right)\right)$, and hence, $h^{\prime}\left(\pi_{1}\left(G^{\prime}\right)\right) / h\left(\pi_{1}(G)\right)$ is a finite (central) subgroup of $\Gamma_{0}(G)$, as desired.

Remark 4.4. Suppose $G$ is semisimple and $G \rightarrow \operatorname{Diff}(M)$ is a nontrivial $G$-action. Then, for some semisimple Lie group $G^{\prime}, M$ admits an effective $G^{\prime}$-action canonically induced by the given $G$-action: simply let $G^{\prime}=G / N$, where $N=$ $\operatorname{ker}(G \rightarrow \operatorname{Diff}(M))$. As $G$ has no nontrivial connected abelian normal subgroups, neither does $G^{\prime}$ and hence $G^{\prime}$ is semisimple.

Suppose $S \subset G$ is a circle subgroup acting nontrivially on $M$. $S \cap N$ must be finite; hence, $S^{\prime}$, the image of $S$ in $G^{\prime}$, is also a circle subgroup, and $M^{S^{\prime}}=M^{S}$.

We will need this observation in some later results.

4.1.2. G-actions on aspherical manifolds and "the like". Suppose $M^{m}$ is such that $f_{*}\left[M^{m}\right] \neq 0 \in H_{m}(\pi ; \mathbb{Q})$. In light of the above discussion, we can gain insight into what Lie group actions $M$ can admit. We first mention two earlier results.

[4] shows that, if $M$ is aspherical, then the only Lie groups acting effectively on $M$ are tori with dimensions bounded above by $\operatorname{rank} \mathcal{Z}(\pi)$ ( " $\mathcal{Z}$ " signifying "the center of"), and such effective torus-actions must be fixed-point-free and have only finite isotropy subgroups. [12] shows that, if there exist $\omega_{1}, \cdots, \omega_{m} \in H^{1}(M)$ such that $\prod_{j} \omega_{j}\left[M^{m}\right] \neq 0$, then the only Lie groups acting effectively on $M$ are tori.

Note that weaker than the hypotheses of both of the above results is the condition that $f_{*}[M] \neq 0$; thus, generalizing them is the next theorem, part (1) of which is observed by [3] and implies the other parts in a way similar to the development of [4.

Theorem 4.5. Let $M^{m}$ be a manifold satisfying $f_{*}[M] \neq 0 \in H_{m}(\pi ; \mathbb{Q})$.

(1) If $S^{1}$ acts nontrivially on $M$ and $o: S^{1} \rightarrow M$ is the orbit of the basepoint in $M$, then $o_{*}: \pi_{1}\left(S^{1}\right) \rightarrow \pi$ is an injection.

(2) If $G$ is semisimple, then $G$ cannot act nontrivially on $M$.

(3) If $G$ acts effectively on $M$, then $G$ is a torus whose dimension is no greater than the rank of $\mathcal{Z}(\pi)$. Hence, if $\pi$ has finite center, then all compact subgroups of Diff $(M)$ are finite. 
(4) If $G$ acts nontrivially on $M$, then, for some $g \in G, M^{g}=\varnothing$ and hence $\chi(M)$ and all Pontrjagin numbers of $M$ vanish. If, in addition, $H^{1}(M)=$ $\{0\}$ then $(P \cdot C)[M]=0$.

(5) If a torus $T$ acts effectively on $M$, then all isotropy subgroups are finite.

Proof. Suppose $S^{1} \stackrel{\gamma}{\rightarrow} \operatorname{Diff}(M)$ is a nontrivial action. Then $\operatorname{ker} \gamma \subset S^{1}$ is finite, and Corollary 4.3 applies. If $o_{*}\left(\pi_{1}\left(S^{1}\right)\right)$ were finite, then Corollary 4.2 would give the following commutative diagram:

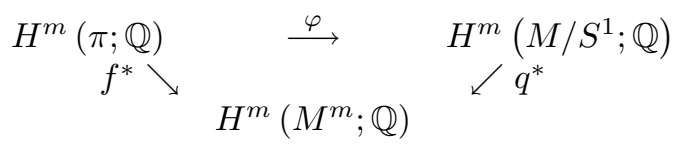

As the action is nontrivial, $\operatorname{dim} M / S^{1}=m-1$; hence, $H^{m}\left(M / S^{1} ; \mathbb{Q}\right)=\{0\}$. But by hypothesis,

$$
0 \neq f^{*}: H^{m}(\pi ; \mathbb{Q}) \longrightarrow H^{m}(M ; \mathbb{Q}) .
$$

This contradiction shows that $o_{*}\left(\pi_{1}\left(S^{1}\right)\right)$ must be infinite; as $\pi_{1}\left(S^{1}\right) \simeq \mathbb{Z}$, $o_{*}$ is injective. This proves (1).

Notation. In the remainder of the proof, whenever $G$ acts nontrivially on $M$, let $S \subset G$ be some circle subgroup of $G$ that also acts nontrivially on $M$.

For (2), suppose the contrary. Let $o: S \rightarrow M$ be the $S$-orbit of the basepoint in $M$. Then $o$ factors into $S \hookrightarrow G \stackrel{o^{\prime}}{\rightarrow} M, o^{\prime}$ being the $G$-orbit map. As $\pi_{1}(G)$ is finite, $o_{*}\left(\pi_{1}(S)\right)$ must also be finite, contradicting (1).

(3) follows from (2) and the structure of a compact connected Lie group $G$ :

$$
G \simeq\left(T \times \prod_{i=1}^{N} H_{i}\right) / \mathcal{C}
$$

where $T$ is a torus, $H_{i}$ are simple Lie groups, and $\mathcal{C}$ is a finite central subgroup of the product. Suppose $G \stackrel{\gamma}{\rightarrow} \operatorname{Diff}(M)$ is an effective action. If $G$ is not toral, then, each $H_{j}$ would act nontrivially on $M$ via

$$
H_{j} \hookrightarrow\left(T \times \prod H_{i}\right) \longrightarrow G \stackrel{\gamma}{\longrightarrow} \operatorname{Diff}(M),
$$

contradicting (2). Hence, $G=T^{n}$. To see that $n \leq \operatorname{rank} \mathcal{Z}(\pi)$, we let $o: T^{n} \rightarrow M$ be the $T^{n}$-orbit and show that $o_{*}: \pi_{1}\left(T^{n}\right) \rightarrow \pi$ is injective, which will yield the desired inequality. We simply note that a nontrivial element in ker $o_{*}$ can be realized as a nontrivial Lie group homomorphism $S^{1} \stackrel{\alpha}{\rightarrow} T^{n}$, which gives rise to a nontrivial $S^{1}$-action on $M$ via $S^{1} \stackrel{\alpha}{\rightarrow} T^{n} \stackrel{\gamma}{\rightarrow}$ Diff $(M)$. o $\alpha$ is then the $S^{1}$-orbit. But $(o \alpha)_{*}\left[\operatorname{Id}_{S^{1}}\right]=o_{*}[\alpha]=0$ by the choice of $\alpha$. Hence, $\pi_{1}\left(S^{1}\right) \stackrel{(o \alpha)_{*}}{\longrightarrow} \pi$ is then trivial, contradicting $(1)$.

For (4), let $g \in S$ be a topological generator of $S$. Then, $M^{S}=M^{g}$. If $M^{S} \neq \varnothing$, by picking a fixed point as the basepoint, the orbit map $S \rightarrow M$ is constant, contradicting (1). The vanishing of the Euler characteristic and Pontrjagin numbers follows readily. With the condition that $H^{1}(M)=\{0\}$, Theorem 3.11 implies the vanishing of $(P \cdot C)[M]$.

(5) also follows from (1). For $a \in M$, let $T_{a}$ be the isotropy subgroup of $a$. If $T_{a}$ were infinite, then its identity component would contain a circle group acting effectively on $M$ fixing $a$, contradicting (1). 
A similar argument would show

Proposition 4.6. Suppose $M$ is a manifold with an effective $G$-action having only finite isotropy groups. Let

$$
d=\max \left\{j \mid 0 \neq f^{*}: H^{j}(\pi ; \mathbb{Q}) \longrightarrow H^{j}(M ; \mathbb{Q})\right\}
$$

If either $G$ is semisimple or $\mathcal{Z}(\pi)$ is finite, then $\operatorname{dim} G \leq \operatorname{dim} M-d$.

Proof. When $G$ acts on $M$ with finite isotropy groups, $M / G$ is a rational homology manifold with $\operatorname{dim} M / G=\operatorname{dim} M-\operatorname{dim} G$. As either $G$ is semisimple or $\mathcal{Z}(\pi)$ is finite, Corollary 4.2 applies. If $\operatorname{dim} G>\operatorname{dim} M-d$, then $H^{d}(M / G ; \mathbb{Q})=\{0\}$ and hence $0=f^{*}: H^{d}(\pi ; \mathbb{Q}) \rightarrow H^{d}(M ; \mathbb{Q})$, which is contrary to the hypothesis.

4.2. Higher vanishing theorems. We now turn to the main results of the present section.

First we introduce a lemma implicit in 3 .

A $G$-submanifold $K$ in a $G$-manifold $M$ is said to be transverse if $K$ meets $M^{H}$ transversally for every closed subgroup $H \subset G$.

Lemma $4.7([3])$. Let $M$ be a $G$-manifold. For each nonzero

$$
y \in \operatorname{Im}\left(H^{l}(M / G) \rightarrow H^{l}(M / G ; \mathbb{Q})\right)
$$

there exists an l-codimensional framed transverse $G$-submanifold $\iota: K_{y} \hookrightarrow M$ such that $q^{*}(y) \frown[M]=\iota_{*}\left[K_{y}\right]$ where $q: M \rightarrow M / G$ is the quotient map.

We are now ready to state the main results.

Theorem 4.8. Suppose $M^{m}$ admits a nontrivial action by a semisimple Lie group $G$ such that there exists a circle subgroup $S \subset G$ with $M^{S}=M^{G}$. Let $x \in$ $H^{m-2 n}(\pi ; \mathbb{Q})$ be a nonzero class such that

$$
\varphi(x) \in \operatorname{Im}\left(H^{m-2 n}(M / G) \rightarrow H^{m-2 n}(M / G ; \mathbb{Q})\right)
$$

where

$$
\varphi: H^{m-2 n}(\pi ; \mathbb{Q}) \rightarrow H^{m-2 n}(M / G ; \mathbb{Q})
$$

is as in Corollary 4.2. Then, $\left(f^{*}(x) \cdot P \cdot C\right)[M]$ can be determined in terms of $K_{\varphi(x)}^{S} \stackrel{\iota}{\hookrightarrow} K_{\varphi(x)}$ and the $S$-action on the normal bundle of $K_{\varphi(x)}^{S} \hookrightarrow K_{\varphi(x)}$, where $K_{\varphi(x)}$ is as in Lemma 4.7 .

Proof. By Remark 4.4, we may assume that the action is effective; by Remark 3.2 and Corollary 4.3 , we may assume that $G$ is simply connected.

If $n=0$, i.e., if $x \in H^{m}(\pi ; \mathbb{Q})$, then, by Theorem $4.5(2), f^{*}(x)[M]=x\left(f_{*}[M]\right)=$ 0 . So assume $n>0$.

Since $K_{\varphi(x)} \hookrightarrow M$ is a submanifold with trivial normal bundle, we identify $\iota^{*}\left(p_{i}\right)$ with $p_{i}\left(K_{\varphi(x)}\right)$. With this in mind and using Corollary 4.2 and Lemma 4.7, we 
compute:

$$
\begin{aligned}
& \left(f^{*}(x) \cdot P\left(p_{1}, \cdots, p_{[m / 4]}\right) \cdot C\right)[M] \\
& =\left(q^{*} \varphi(x) \cdot P \cdot C\right)[M] \\
& =(P \cdot C)\left(q^{*} \varphi(x) \frown[M]\right) \\
& =(P \cdot C) \iota_{*}\left[K_{\varphi(x)}\right] \\
& =\iota^{*}(P \cdot C)\left[K_{\varphi(x)}\right] \\
& =\left(P\left(\iota^{*}\left(p_{1}\right), \cdots, \iota^{*}\left(p_{[m / 4]}\right)\right) \cdot \iota^{*}(C)\right)\left[K_{\varphi(x)}\right] \\
& =\left(P\left(p_{1}\left(K_{\varphi(x)}\right), \cdots, p_{[m / 4]}\left(K_{\varphi(x)}\right)\right) \cdot \iota^{*}(C)\right)\left[K_{\varphi(x)}\right] .
\end{aligned}
$$

Since $K_{\varphi(x)}$ is a $G$-submanifold of $M$ and $M^{S}=M^{G}$, we have $K_{\varphi(x)}^{S}=K_{\varphi(x)}^{G}$. Applying Theorem 3.6 to $K_{\varphi(x)}$ yields the desired result.

Corollary 4.9. If $M^{m}$ admits an action by a semisimple Lie group $G$ such that, for some $g \in G, M^{g}=\varnothing$, then $\left(f^{*}(x) \cdot P \cdot C\right)[M]=0$.

Proof. Just as in the proof of Corollary 3.7, we may assume, without any loss, that $g$ generates a circle subgroup $S \subset G$. Hence, $M^{S}=\varnothing=M^{G}$. Applying Theorem 4.8 yields the desired conclusion.

In the following, for an $S^{1}$-manifold $M$, let $o: S^{1} \rightarrow M$ be the orbit of the basepoint and $o_{*}: \pi_{1}\left(S^{1}\right) \rightarrow \pi_{1}(M)$ the induced map.

Theorem 4.10. Let $M^{m}$ be a fixed-point-free $S^{1}$-manifold and $x \in H^{*}(\pi ; \mathbb{Q})$.

(1) Suppose $\operatorname{Im} o_{*}$ is finite. Then $\left(f^{*}(x) \cdot P\right)[M]=0$; if, in addition, $H^{1}(M)$ $=\{0\}$, then $\left(f^{*}(x) \cdot P \cdot C\right)[M]=0$.

(2) Suppose, for some semisimple Lie group $G$, the $S^{1}$-action extends to a $G$ action, then $\left(f^{*}(x) \cdot P \cdot C\right)[M]=0$.

Proof. As noted earlier, under the hypotheses of both parts of the theorem, for $x \in H^{m}(\pi ; \mathbb{Q}), f^{*}(x)[M]=x\left(f_{*}[M]\right)=0$ by Theorem 4.5(2). So assume $x \in$ $H^{m-2 n}(\pi ; \mathbb{Q})$ with $n>0$; also assume without loss of generality that $x$ satisfies the condition in Theorem 4.8.

A nontrivial action $S^{1} \rightarrow \operatorname{Diff}(M)$ always has a finite kernel, making Corollary 4.3 applicable; finiteness of $\operatorname{Im} o_{*}$ makes Corollary 4.2 applicable. A computation similar to that in (4.1) yields

$$
\begin{aligned}
& \left\{f^{*}(x) \cdot P\left(p_{1}, \cdots, p_{[m / 4]}\right)\right\}[M] \\
& =\left\{q^{*} \varphi(x) \cdot P\left(p_{1}, \cdots, p_{[m / 4]}\right)\right\}[M] \\
& =P\left(p_{1}\left(K_{\varphi(x)}\right), \cdots, p_{[m / 4]}\left(K_{\varphi(x)}\right)\right)\left[K_{\varphi(x)}\right]
\end{aligned}
$$

where $\varphi: H^{m-2 n}(\pi ; \mathbb{Q}) \rightarrow H^{m-2 n}\left(M / S^{1} ; \mathbb{Q}\right)$ is as in Corollary 4.2 and $K_{\varphi(x)} \stackrel{\iota}{\hookrightarrow} M$ is as in Lemma 4.7. As $K_{\varphi(x)}^{S^{1}}=K_{\varphi(x)} \cap M^{S^{1}}, K_{\varphi(x)}^{S^{1}}=\varnothing$; hence Remark 3.10 shows

$$
P\left(p_{1}\left(K_{\varphi(x)}\right), \cdots, p_{[m / 4]}\left(K_{\varphi(x)}\right)\right)\left[K_{\varphi(x)}\right]=0,
$$

proving the first statement of (1).

For the second statement of (1), we note, as in (4.1),

$$
\begin{aligned}
& \left(f^{*}(x) \cdot P\left(p_{1}, \cdots, p_{[m / 4]}\right) \cdot C\right)[M] \\
& =\left(P\left(p_{1}\left(K_{\varphi(x)}\right), \cdots, p_{[m / 4]}\left(K_{\varphi(x)}\right)\right) \cdot \iota^{*}(C)\right)\left[K_{\varphi(x)}\right] .
\end{aligned}
$$


The condition $H^{1}(M)=\{0\}$ makes Corollary 2.2 applicable. Hence, for $C=\prod_{j} c_{j}$ where $c_{j} \in \operatorname{Im}\left(H^{2}(M) \rightarrow H^{2}(M ; \mathbb{Q})\right)$, the $S^{1}$-action on $M$ admits a lifting to an action on each complex line bundle $\mathcal{L}\left(c_{j}\right)$, from which the bundle $\iota^{*} \mathcal{L}\left(c_{j}\right)$ inherits an $S^{1}$-equivariant structure. Applying the proof of Theorem 3.6 to the $S^{1}$-manifold $K_{\varphi(x)}$ yields the result.

(2) is immediate from Corollary 4.9.

When $\operatorname{Im} o_{*}$ is infinite, we may resort to the next theorem, which can be shown using Lemma 4.1 and following the proof of the last theorem.

Theorem 4.11. Suppose $M$ is a fixed-point-free $S^{1}$-manifold. Let $\pi^{\prime}=\pi / \operatorname{Im} o_{*}$, let $\rho: \pi \rightarrow \pi^{\prime}$ be the quotient map, and let $y \in H^{*}\left(\pi^{\prime} ; \mathbb{Q}\right)$. Then, $\left(f^{*} \rho^{*}(y) \cdot P\right)[M]=$ 0 ; if, in addition, $H^{1}(M)=\{0\}$, then, $\left(f^{*} \rho^{*}(y) \cdot P \cdot C\right)[M]=0$.

\section{VANISHING THEOREMS ON SPIN $G$-MANIFOLDS}

We study obstructions to certain $G$-actions on spin manifolds.

If $M$ is a spin manifold admitting a nontrivial $S^{1}$-action, then 1 shows that its $\hat{\mathbf{A}}$-genus vanishes; 3 proves the vanishing of certain "higher $\hat{\mathbf{A}}$-genera". If $M$ is a spin manifold admitting a spin-structure-preserving action by a semisimple Lie group $G$, then, as a consequence of [3]'s result, all "higher $\hat{\mathbf{A}}$-genera" vanish; similar vanishing theorems hold for finer invariants with values in $K$-theory, such as $\hat{\mathcal{A}}$ and $G-\hat{\mathcal{A}}$; see [9] and [1] for details.

In this section, we show some similar results.

As before, let $\pi=\pi_{1}(M)$, let $f: M \rightarrow K(\pi, 1)$ classify the universal cover of $M$, let $x \in H^{*}(\pi ; \mathbb{Q})$, and let $C$ be an element of the subalgebra of $H^{*}(M ; \mathbb{Q})$ generated by $H^{2}(M ; \mathbb{Q})$.

Theorem 5.1. Let $G$ be a semisimple Lie group acting on a spin manifold $M^{2 n}$. Suppose there exists a circle subgroup $S \subset G$ acting nontrivially on $M$ with every component of $M^{S}$ containing a point of $M^{G}$. Then $(\hat{\mathbf{A}}(M) \cdot C)[M]=0$.

Proof. By Remark 3.2, we assume, without loss of generality, that $G$ is simply connected, and $G$ acts isometrically on $M$. As $G$ is now assumed to be simply connected, the $G$-action necessarily preserves the spin structure on $M$.

In the following, we adopt the same notation as in the proof of Theorem 3.1.

Let $c \in \operatorname{Im}\left(H^{2}(M) \rightarrow H^{2}(M ; \mathbb{Q})\right)$. By the $G$-spin Theorem 2.8, for $z$ a topological generator of $S$,

$$
\begin{aligned}
& \operatorname{Spin}_{G}(M ; \mathcal{L}(c))(z) \\
& =\operatorname{Spin}_{S}(M ; \mathcal{L}(c))(z) \\
& =\sum_{\nu}\left\{\sigma_{\nu} z^{l_{\nu}} e^{i_{\nu}^{*}(c)} \hat{\mathbf{A}}\left(M_{\nu}^{S}\right) \prod_{k \in \mathbb{N}} \prod_{j=1}^{d_{\nu}(k)} \frac{z^{\frac{k}{2}} e^{-\frac{1}{2} x_{\nu, j}(k)}}{1-z^{k} e^{-x_{\nu, j}(k)}}\right\}\left[M_{\nu}^{S}\right],
\end{aligned}
$$

$\operatorname{Spin}_{S}(M ; \mathcal{L}(c))(z) \in R(S)$; it is thus a Laurent polynomial $w(z)$. As explained in the proof of Theorem 3.1, $l_{\nu}=0$. Therefore, by the above formula, $w(0)=0=$ 
$w(\infty)$. Hence, $w(z)=0$ for all $z \in \mathbb{C}$. By Theorem 2.9,

$$
\begin{aligned}
0 & =w(1) \\
& =2^{n}(\operatorname{ch}(\mathcal{L}(c)) \hat{\mathbf{A}}(M))[M] \\
& =2^{n}\left(e^{c} \cdot \hat{\mathbf{A}}(M)\right)[M] .
\end{aligned}
$$

It follows then, just as in the proof of Theorem 3.1, that $\left(\hat{\mathbf{A}}(M) \cdot c^{d}\right)[M]=0$. Using Lemma 3.4 yields the desired result.

Using techniques of $\S 4$, this result can be modified to produce an obstruction from the fundamental group.

Theorem 5.2. Let $G$ be a semisimple Lie group acting on a spin manifold $M^{m}$. Suppose there exists a circle subgroup $S \subset G$ acting nontrivially on $M$ with $M^{S}=$ $M^{G}$. Then, $\left(f^{*}(x) \cdot \hat{\mathbf{A}}(M) \cdot C\right)[M]=0$.

Proof. Let $x \in H^{m-2 n}(\pi ; \mathbb{Q})$. If $n=0$, then, by Theorem $4.5(2), f^{*}(x)[M]=$ $x\left(f_{*}[M]\right)=0$. So assume $n>0$. Also assume, without loss of generality, that $x$ satisfies the condition in Theorem 4.8. As in (4.1),

$$
\begin{aligned}
& \left(f^{*}(x) \cdot \hat{\mathbf{A}}(M) \cdot C\right)[M] \\
& =\left(q^{*} \varphi(x) \cdot \hat{\mathbf{A}}(M) \cdot C\right)[M] \\
& =(\hat{\mathbf{A}}(M) \cdot C)\left(q^{*} \varphi(x) \frown[M]\right) \\
& =(\hat{\mathbf{A}}(M) \cdot C) \iota_{*}\left[K_{\varphi(x)}\right] \\
& =\iota^{*}(\hat{\mathbf{A}}(M) \cdot C)\left[K_{\varphi(x)}\right] \\
& =\left(\hat{\mathbf{A}}\left(K_{\varphi(x)}\right) \cdot \iota^{*}(C)\right)\left[K_{\varphi(x)}\right] .
\end{aligned}
$$

Since $K_{\varphi(x)}$ has trivial normal bundle in a spin manifold $M, K_{\varphi(x)}$ is also a spin manifold; as $K_{\varphi(x)}$ is a transverse $G$-submanifold, the $S$-action on it is nontrivial. Hence, Theorem 5.1, applied to $K_{\varphi(x)}$, yields the result.

\section{REFERENCES}

[1] M. Atiyah and F. Hirzebruch, Spin-Manifolds and Group Actions, Essays on Topology and Related Topics, 18-28, Springer-Verlag, New York, Heidelberg, Berlin, 1970. MR0278334 (43:4064)

[2] M. Atiyah and I. M. Singer, The Index of Elliptic Operators, III, Ann. of Math. 87 (1968), 546-604. MR0236952(38:5245)

[3] W. Browder and W.-C. Hsiang, G-actions and the Fundamental Group, Invent. Math. 65 (1982), 411-424. MR0643560 (83d:57026)

[4] P. Conner and F. Raymond, Actions of Compact Lie Groups on Aspherical Manifolds, Topology of Manifolds (edited by J. C. Cantrell and C. H. Edwards, Jr.), 227-264, Markham, Chicago, 1970. MR0271958 (42:6839)

[5] F. Fang and X. Rong, Fixed Point Free Circle Actions and Finiteness Theorems, Commun. in Contemp. Math. 2, no. 1 (2000), 75-86. MR1753140(2001d:53044)

[6] Y. Guillemin and S. Sternberg, Supersymmetry and Equivariant de Rham Theory, Math. Past and Present, Vol. 2, Springer-Verlag, Berlin, Heidelberg, New York, 1999. MR 1689252 (2001i:53140) 
[7] W. Y. Hsiang, Cohomology Theory of Topological Transformation Groups, Ergebnisse der Math. und ihrer Grenzgebiete, Band 85, Springer-Verlag, New York, Heidelberg, Berlin, 1975. MR0423384 (54:11363)

[8] R. K. Lashof, P. May and G. B. Segal, Equivariant Bundles with Abelian Structure Group, Contemp. Math., Vol. 19, 167-176, AMS, Providence, R.I., 1983. MR.0711050 (85b:55023)

[9] H. B. Lawson Jr., and S.-T. Yau, Scalar Curvature, Non-Abelian Group Actions and the Degree of Symmetry of Exotic Spheres, Comm. Math. Helv., Vol. 49, (1974), 232-244. MR0358841 (50:11300)

[10] J. McCleary, A User's Guide to Spectral Sequences (2nd Ed.), Cambridge Studies in Advanced Math., Vol. 58, Cambridge University Press, 2001. MR1793722 (2002c:55027)

[11] J. Rosenberg and S. Weinberger, Higher G-indices and applications, Ann. Ec. Norm. Sup (4) 21, (1988), 479-495. MR0982331 (90f:58170)

[12] S. T. Yau, Remarks on the Group of Isometries of a Riemannian Manifold, Topology 16 (1977), 239-247. MR0448379(56:6686)

Department of Mathematics, Indiana University, Bloomington, Indiana 47405

E-mail address: pding@indiana.edu 